\title{
Transient Interaction between a Reaction Control Jet and a Hypersonic Crossflow
}

\author{
Warrick A. Miller and Paul R. Medwell \\ School of Mechanical Engineering, University of Adelaide, South Australia, 5005, Australia.* \\ Con J. Doolan \\ School of Mechanical and Manufacturing Engineering, \\ University of New South Wales, New South Wales, 2052, Australia \\ Minkwan Kim \\ Astronautics Research Group, University of Southampton, Southampton SO17 1BJ, UK
}

(Dated: February 11, 2018)

This paper presents a numerical study that focuses on the transient interaction between a reaction control jet and a hypersonic crossflow with a laminar boundary layer. The aim is to better understand the underlying physical mechanisms affecting the resulting surface pressure and control force. Implicit large-eddy simulations were performed with a round, sonic, perfect air jet issuing normal to a Mach 5 crossflow over a flat plate with a laminar boundary layer, at a jet-to-crossflow momentum ratio of 5.3 and a pressure ratio of 251 . The pressure distribution induced on the flat plate is unsteady and is influenced by vortex structures that form around the jet. A horseshoe vortex structure forms upstream, and consists of six vortices: two quasi-steady vortices and two co-rotating vortex pairs that periodically coalesce. Shear-layer vortices shed periodically and cause localised high pressure regions that convect downstream with constant velocity. A longitudinal counter-rotating vortex pair is present downstream of the jet, and is formed from a series of trailing vortices which rotate about a common axis. Shear-layer vortex shedding causes periodic deformation of barrel and bow shocks. This changes the location of boundary layer separation which also affects the normal force on the plate.

\footnotetext{
*warrick.miller@adelaide.edu.au.
} 


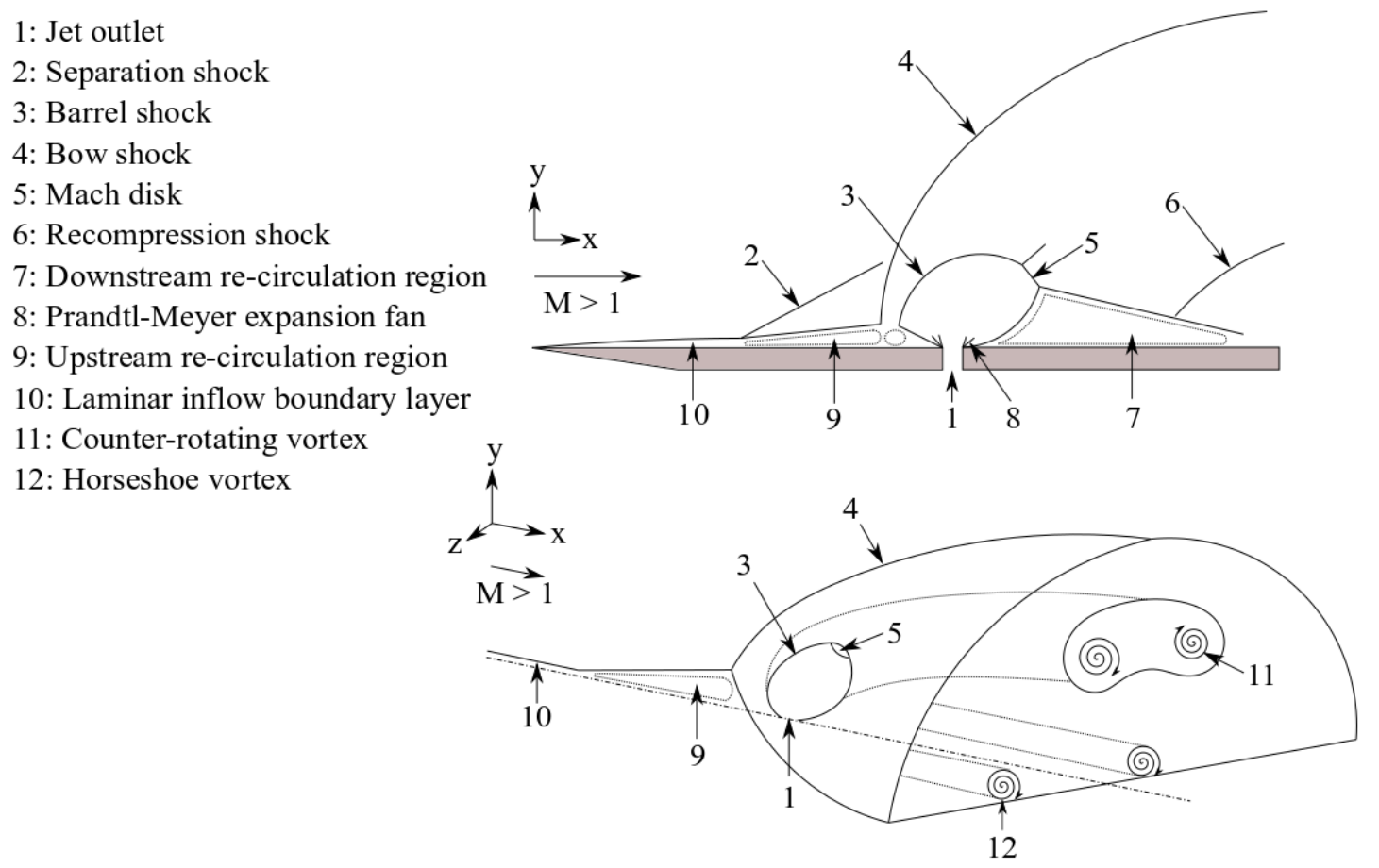

FIG. 1: Schematic of the two- and three-dimensional time-averaged interaction flow-field.

\section{INTRODUCTION}

Air-breathing hypersonic vehicles are typically designed with an integrated scramjet engine, and can exhibit unique dynamic characteristics $[1,2]$ that require high bandwidth control, beyond what may be normally provided by conventional aerodynamic control surfaces. Reaction control jets are an alternative actuation method to increase the control system bandwidth, as they can provide large forces and operate at high frequency.

Reaction control jets generate actuation force through two mechanisms: the thrust of the jet and the interaction between the jet and crossflow, caused by induced pressure distribution on the surface of the vehicle. The jet-incrossflow interaction is complex, unsteady, and can significantly affect the total control force. The surface pressure distribution is also important when considering sonic fatigue and supersonic panel flutter near fuel injectors in scramjet engines [3].

The physics controlling the interaction force between a sonic, under-expanded jet and supersonic crossflow has been the subject of a large body of research $[4,5]$. Early experimental studies determined various aspects of the time-averaged flow structure, including shock shape and location, jet concentration profiles, geometry of separated flow regions, and pressure fields [6-8]. A schematic of the typical time-averaged shock and vortex structure is shown in Figure 1. The shock structure consists of a bow shock (4) and re-circulation region ahead of the jet (9), a PrandtlMeyer expansion fan centred at the edge of the jet nozzle (8), barrel shocks (3) and a Mach disk (5). A single horseshoe vortex (12) is present near the plate and wraps around the base of the jet [9], while a counter-rotating vortex pair (11) dominates the far-field [10]. While the time-averaged flow-field is well documented, these structures exhibit significant temporal variations.

In the unsteady flow, horseshoe vortices form upstream of the jet leading edge in the re-circulation region. In a subsonic crossflow, Kelso et al. [11] observed that the downstream structure of the horseshoe vortex depends on the sign of vorticity. Horseshoe vortices with vorticity the same sign as the wall boundary layer extend downstream along the flat plate and are incorporated into the wake. Horseshoe vortices with the opposite sign to the boundary layer are lifted from the wall. Kelso et al. [11] also observed that horseshoe vortices in subsonic crossflow fall into one of three regimes: steady, oscillating, or coalescing. The specific regime determines whether two or three distinct vortices are present. Oscillations and periodicity were observed by Krothapalli et al. [12] at certain jet-to-crossflow velocity ratios, while several other studies have shown a steady horseshoe vortex [13]. The horseshoe vortex regimes influence the unsteady surface pressure upstream of the jet outlet, and therefore will influence the control force.

In a supersonic crossflow, Viti et al. [14] observed horseshoe vortex behaviour similar to that of a subsonic crossflow. Fluid from the re-circulation region with vorticity the opposite sign to the wall boundary layer was transported from 
the wall and deposited behind the jet to form part of the counter-rotating vortex pair, while fluid from vortices with the same sign as the wall boundary layer travelled downstream along the plate. Additional vortices within the recirculation region have been reported in other studies [15], which behave in a similar manner, depending on the sign of vorticity.

There is another large re-circulation region downstream of the jet outlet, where the flow remains separated from the plate. In this region the time-averaged velocity field is dominated by a single streamwise-oriented counter-rotating vortex pair [10]. The single counter-rotating vortex pair observed in the time-averaged flow emerges from the merging of three smaller longitudinal trailing vortices [14]; two vortices are developed by contact between the slow jet fluid downstream of the Mach disk and the fast freestream, the other originates on the windward side of the barrel shock. These three vortices rotate with respect to each other around a common longitudinal axis. While the existence of these vortices has been reported [14], their unsteady motion has not been detailed. As these vortices dominate the far-field, their unsteady motion is likely to have a significant influence on the interaction force. Other longitudinal vortices have been identified $[9,14,16]$, the structure of which depends on freestream Mach number.

Several structures are present in the unsteady jet-in-crossflow interaction that are not observed in the time-averaged flow-field. Jet shear-layer vortices form at the upstream barrel shock, and are shed periodically due to KelvinHelmholtz instability in the shear-layer. The behaviour of these vortices depends on jet-to-crossflow velocity ratio, $R=U_{j} / U_{\infty}$; jet pressure, $p_{j}$; jet Reynolds number, $R e_{d j}=U_{j} d_{j} / \nu_{j}$; and jet pipe geometry [17]. Several authors $[11,18-20]$ have proposed different mechanisms for the development and evolution of jet shear-layer vortices in a subsonic crossflow. Smith and Mungal [21] observed that the formation of the jet shear-layer vortices is delayed in a subsonic crossflow with increased velocity ratios $R>5$. Recent work in subsonic crossflows shows that this delayed formation is not related to velocity ratio, but rather to shear-layer instabilities [22-24]. There are two distinct mechanisms for jet shear-layer roll-up in subsonic crossflow. For jets with absolutely unstable shear-layers, there is a rapid, or immediate shear-layer vorticity roll-up. This corresponds to formation of a clear, symmetric counter-rotating vortex pair [24]. If the shear-layer is convectively unstable, the shear-layer vorticity roll-up is delayed. This can result in an asymmetric, or non-existent vortex-pair in the mean jet cross-section. At successively lower momentum ratio, the location of shear-layer roll-up moves closer to the jet exit and the spacing between individual vortices is reduced [24]. This information allows significant increases in penetration and mixing to be achieved through tailored pulsing schemes [23].

The evolution of shear-layer vortices in supersonic crossflow is more complex than subsonic crossflow, although the structures are qualitatively similar [25]. Large variations in size, shape, symmetry and periodicity of shear-layer vortices were observed by van Lerberghe et al. [26]. Kawai and Lele [16] reported periodic shear-layer vortex shedding at Strouhal Numbers, $S t_{\infty}=0.4-0.6$, based on jet diameter and free-stream velocity, $S t_{\infty}=f \times d_{j} / U_{\infty}$, in a Mach 1.6 crossflow, with a jet-to-crossflow momentum ratio, $J=\rho_{j} U_{j}^{2} / \rho_{\infty} U_{\infty}^{2}$, of 1.7 and a turbulent in-flow boundary layer. Chai et al. [25] reported a dominant global mode at $S t_{\infty}=0.3$ at the same conditions. Ben-Yakar [27] reported a shedding frequency of $S t_{j}=1$, based on jet diameter and jet outlet velocity, $S t_{j}=f \times d_{j} / U_{j}$, for a higher Mach number $(M)$ flow with higher momentum ratio $\left(M_{\infty}=3.4, J=4.1\right)$; while Andre et al. [28] investigated the development of shear-layer vortices for a range of pressure ratios in a Mach 4.2 crossflow.

In a supersonic crossflow, when jet velocity is low compared to free-stream velocity (i.e. $R<2$ ), shear-layer vortices take the form of hairpin vortices, rather than vortex rings [29]. As a result, the shear-layer vortices are sometimes referred to as hairpin vortices in this regime [28]. These vortices are convected downstream at a velocity approaching the free-stream velocity. Gruber et al. [30] tracked the development and convection of jet shear-layer vortices for circular and elliptical jets, using both air and helium, in a Mach 2 crossflow, at $J=1.9$. Similar studies have been conducted [31-34] to track shear-layer vortex convection, stretching and tilting. Differences in convective and mixing properties have been observed for different injectant species. Shear-layer vortices were found to persist long distances for hydrogen jets, while ethylene jets tended to break down nearer the jet exit.

Wake vortices are unsteady upright tornado-like vortices present immediately downstream of the jet exit. Fric and Roshko [17] suggest that wake vortices originate in the boundary layer and terminate in the counter-rotating vortex pair. However, jet fluid has also been observed to flow into the boundary layer in subsonic crossflow, for velocity ratios $5<R<32$ [5]. In comparison to subsonic crossflow, wake vortices formed in a supersonic crossflow tend not to be organised, and are much less distinct than the shear-layer vortices [26]. As such, their influence on the interaction force is likely to be small.

The shock structure also contains a number of unsteady features. Unsteadiness has been observed in the barrel shock region, most visible as a periodic flattening of the windward barrel shock into two or more straight segments that join at sharp corners [26,35]. At other times, indentations or rippling was also observed in the bow shock, with little or no shock movement. Kawai et al. [16] identified the source of both the periodic flattening of the barrel shock and deformation of the bow shock as pressure fluctuations inside the upstream re-circulation region. Kawai et al. [16] also identified a set of counter-rotating vortices inside the boundary layer in the wake region, and proposed that these were induced by suction from the counter-rotating vortex pair. Viti et al. [14] contend that these vortices originate in 
the re-circulation region immediately upstream of the jet outlet. Viti et al. [14] also found that, in a Mach 4 crossflow, there is an upper set of counter-rotating vortices, that follow the leading edge of the bow shock away from the plate.

Although it is critical to the formation of the high pressure region upstream of the jet outlet that augments the control force, investigations into the unsteady shear-layer vortex structure for hypersonic $(M>5)$ crossflows with a laminar boundary layer have not been reported. Chai et al. [25] and Kawai and Lele [36] considered a laminar in-flow boundary layer at the same flow conditions as Santiago and Dutton [35] $\left(M_{\infty}=1.6, J=1.7\right)$. With the boundary layer thickness matched to the experimental (turbulent) boundary layer, the shear-layer vortex shedding is reduced from $S t_{\infty}=0.4-0.6$ to $S t_{\infty}=0.2$, and the upstream and downstream re-circulation regions are larger, driving significant differences in wall pressure distributions. The influence of the thicker laminar in-flow boundary layer expected at higher free-stream Mach number has not been reported, and is addressed in the present study.

In addition, while significant attention has been paid to the shear-layer vortices, the unsteady motion of horseshoe vortices in the upstream re-circulation region and the longitudinal trailing vortices downstream has not been detailed in a supersonic crossflow with a laminar boundary layer.

The aim of this study is to use numerical simulations to extend the previous work by considering the interaction of a circular jet with a hypersonic $\left(M_{\infty}=5\right)$ crossflow over a flat plate with a laminar boundary layer, at an increased jet-to-crossflow momentum ratio $(J=5.3)$. A detailed understanding of the unsteady and time-averaged behaviour of shock and vortex structures in this regime is required as the behaviour of these structures influence the control force produced by a reaction control jet, as well as the dynamic loading of surface panels. Therefore, a thorough understanding of these phenomena will improve estimates of the jet actuation force. Numerical simulation will be used to characterise the temporal motion of structures which affect the time dependent control force caused by a reaction control jet operating in a hypersonic crossflow with a laminar boundary layer. The temporal evolution of the horseshoe vortices, shear-layer vortices, trailing vortices and wake vortices will be detailed, and their influence on the surface pressure visualised. The motion of the shock structure, consisting of a separation shock, bow shock, barrel shocks, Mach shock and downstream recompression shock will also be visualised, and the impact of this motion on the surface pressure will be shown.

\section{COMPUTATIONAL SETUP}

\section{A. Governing equations}

The governing equations for this study are the compressible, unsteady Navier Stokes equations:

$$
\begin{aligned}
\frac{\partial \rho}{\partial t}+\frac{\partial}{\partial x_{i}}\left(\rho u_{i}\right) & =0 \\
\frac{\partial}{\partial t}\left(\rho u_{i}\right)+\frac{\partial}{\partial x_{i}}\left(\rho u_{i} u_{j}+p \delta_{i j}-\boldsymbol{\tau}_{i j}\right) & =0 \\
\frac{\partial}{\partial t}(\rho E)+\frac{\partial}{\partial x_{i}}\left((\rho E+p) u_{i}-q_{i}-u_{j} \boldsymbol{\tau}_{i j}\right) & =0
\end{aligned}
$$

where $\rho$ is density, $u_{i}$ is the flow velocity in the $i$ direction using Einstein notation, $p$ is pressure, $t$ is time, and $q$ is heat flux, defined as follows:

$$
q_{i}=k \frac{\partial T}{\partial x_{i}}
$$

where $T$ is temperature, $k$ is thermal conductivity, defined using a constant specific heat at constant volume, $c_{v}$, and a constant Prandtl number of 0.7 . The total energy density is $E=e+u_{i} u_{i} / 2$ with $e$ the specific internal energy, and $\boldsymbol{\tau}_{i j}$ is the deviatoric component of the viscous stress tensor, assuming a Newtonian fluid for which, using Stokes' hypothesis:

$$
\boldsymbol{\tau}_{i j}=\mu\left[\left(\frac{\partial u_{i}}{\partial x_{j}}+\frac{\partial u_{j}}{\partial x_{i}}\right)-\frac{2}{3} \frac{\partial u_{k}}{\partial x_{k}} \delta_{i j}\right]
$$

where $\mu$ is viscosity, modeled using Sutherland's law. In this work, only perfect gases are considered, for which: 
TABLE I: Initial conditions of the simulation.

\begin{tabular}{cccc|cccc}
\hline \multicolumn{4}{c|}{ Free-stream } & \multicolumn{3}{c}{ Jet } \\
\hline$M_{\infty}$ & $p_{\infty}(\mathrm{Pa})$ & $T_{\infty}(\mathrm{K})$ & $R e\left(\times 10^{6} / \mathrm{m}\right)$ & $M_{j}$ & $p_{0 j} / p_{\infty}$ & $R e_{d j}\left(\times 10^{3}\right)$ & $J$ \\
\hline 5 & 1210 & 62.5 & 13.1 & 1 & 251 & 88.5 & 5.3 \\
\hline
\end{tabular}

$$
\begin{aligned}
& p=\rho R_{s} T \\
& e=c_{v} T=\frac{R_{s}}{(\gamma-1)} T
\end{aligned}
$$

where $R_{s}$ is the specific gas constant and $\gamma$ is the ratio of specific heats.

The equations are solved in dimensional form using the rhoCentralFoam finite volume solver, which forms part of the open-source OpenFOAM code [37]. For compressible flows, fluid properties are not only transported by the flow, but also by the propagation of waves. Thus, the numerical scheme used to solve the governing equations requires the flux interpolation to be stabilised based on transport that can occur in any direction [38]. To achieve this, the central-upwind flux reconstruction method of Kurganov et al. [39] was used, with the van Leer limiter [40]. The second-order, implicit Crank-Nicholson method is employed for temporal discretisation [41].

An implicit large-eddy simulation (LES) methodology has been adopted to model turbulence. Conventional LES relies on a spatial and temporal filtering technique that eliminates fluctuations smaller than a predefined cut-off, determined by the grid size; additional terms are treated via a sub-grid scale (SGS) model. An alternative to SGS models, which attempt to explicitly estimate the sub-grid viscosity, is to use the inherent dissipation of the numerical scheme to provide the required damping. Numerical schemes that utilise flux-limiting schemes implicitly provide a SGS model [42]. This approach is referred to as Monotone Integrated Large Eddy Simulation (MILES). The implicit SGS model inherent in a finite volume discretisation of the compressible Navier-Stokes equations has been derived using modified equations analysis, and is of the eddy-viscosity type [43]. MILES has been used to study a wide range of flow-fields, including channel flows, Kelvin-Helmholtz instability, and vortex ring dynamics [44], and good agreement has been demonstrated between MILES and conventional LES. A thorough review of MILES and its validity for a broad range of applications is provided by Grinstein et al. [45]. Importantly, MILES has shown performance comparable to conventional LES for similar flows, including jets in supersonic crossflow $[9,16,28,44]$, at reduced computational cost. As MILES does not explicitly add dissipation, it can accurately capture a Laminar flow, where conventional LES requires complex dynamic procedures to limit the operation of the sub-grid viscosity to regions of turbulence. It has also been shown that less diffusive limiters, such as the Superbee limiter [46], outperform the more diffusive van Leer limiter in some cases. However, the van Leer limiter is used in this work as it performs better in reducing spurious oscillatory behaviour in the region of shocks with this numerical scheme [47].

The present numerical scheme has been demonstrated to accurately simulate shocks through simulation of canonical flows, including a shock tube, wedge, diamond airfoil, forward step, and jet in quiescent atmosphere [47].

\section{B. Geometric configuration and flow conditions}

The geometric configuration is a sonic jet issuing from a flat plate into a Mach 5, perfect air crossflow. This flow has been investigated experimentally by Erdem [48]. The initial conditions of the simulation are shown in Table I.

A three-dimensional computational domain was generated to approximate the experimental geometry, which is represented schematically in Figure 2. The upstream edge of the domain is a supersonic inlet, placed $10 \mathrm{~mm}$ upstream from the leading edge of the sharp-edged flat plate. A supersonic (Neumann) outlet is placed at the downstream edge of the plate. The plate itself is modelled as an adiabatic wall, with a circular sonic inlet with diameter $d_{j}=2 \mathrm{~mm}$ representing the jet, placed $105 \mathrm{~mm}$ downstream from the leading edge. Geometric symmetry allows the domain to be split in half, with a symmetry condition applied in a plane parallel to the free-stream flow, through the centre-line of the jet. Far-field (Neumann) boundary conditions were placed at the top and side, at a distance of $100 \mathrm{~mm}$ and $35 \mathrm{~mm}$ respectively, to complete the domain. These distances are sufficient to capture all relevant flow structures. The jet was assumed to have a step velocity profile, no boundary layer or inlet plenum was modelled. This is a common simplification when considering choked nozzle flows, and has been shown to have little or no effect on the shock formations in the crossflow $[28,49]$.

The simulation was initialised without the jet flow to allow a steady laminar boundary layer to be established on the plate. The jet was then started by instantaneously applying the pressure, temperature and velocity values shown 


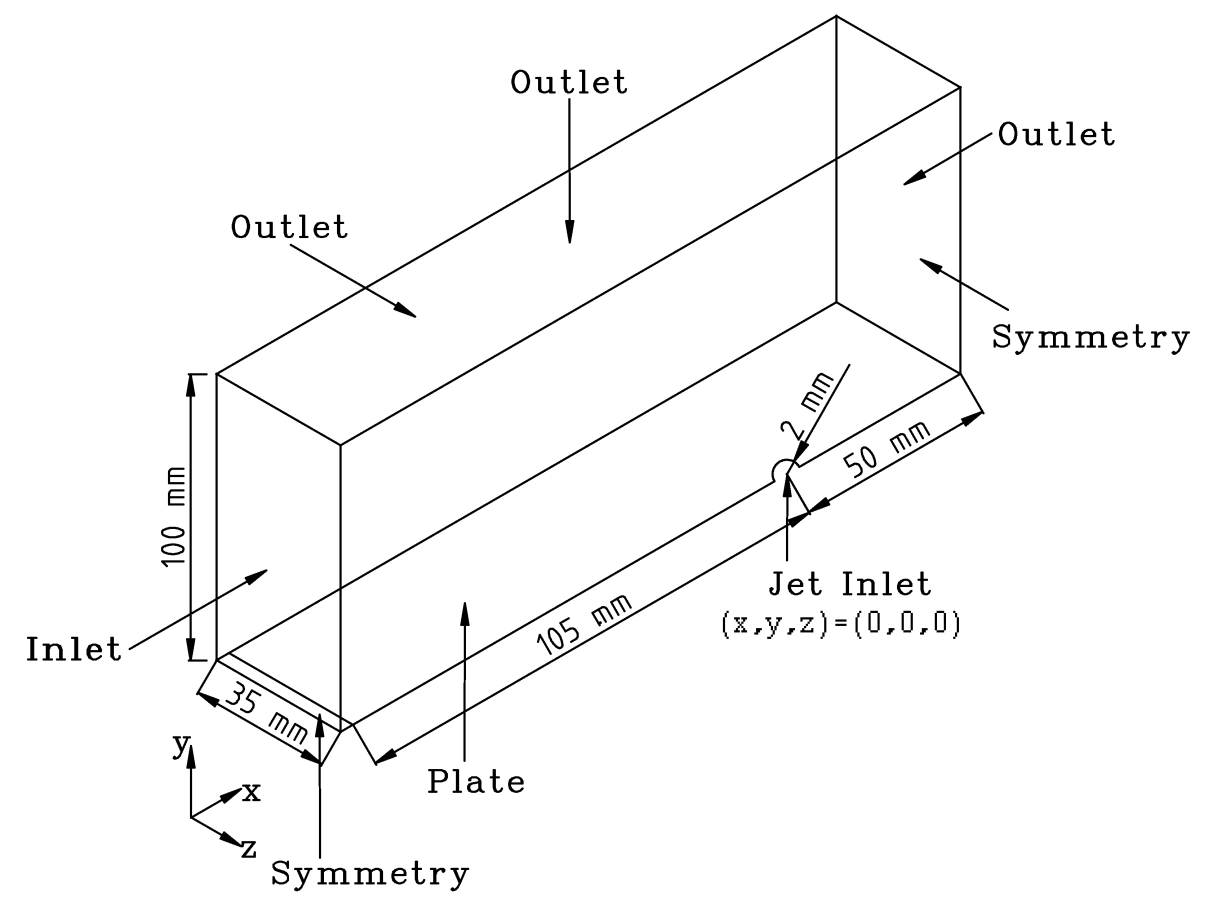

FIG. 2: Schematic of the geometric configuration and flow conditions.

TABLE II: Mesh properties.

\begin{tabular}{lcccc}
\hline Mesh & $\begin{array}{c}N_{\text {cells }} \\
\left(\times 10^{6}\right)\end{array}$ & $\begin{array}{c}\text { Cell size } \\
(\times 1 / \delta)(x, y, z)\end{array}$ & $\begin{array}{c}\text { First cell height } \\
\left(y^{+}\right)\end{array}$ & $\begin{array}{c}\text { Mach disk height } \\
\left(\times d_{j}\right)\end{array}$ \\
\hline Coarse & 1.9 & $(34,34,31)$ & 1.5 & 3.2 \\
Intermediate & 6.4 & $(50,50,49)$ & 0.75 & 3.3 \\
Fine & 15.5 & $(68,68,63)$ & 0.75 & 3.3 \\
\hline
\end{tabular}

in Table I at the jet inlet for an initial start-up period of $\tau=200$, where $\tau$ is a non-dimensional time parameter, defined as:

$$
\tau=\frac{t \times U_{\infty}}{d_{j}}
$$

This corresponds to 2.4 total domain flow-through periods, or 6 times the time taken for the jet flow to reach the outlet, which is sufficient for the normal force on the plate to reach a quasi-steady state. After this initial start-up period, time-averaged results were obtained over approximately 5 domain flow-through periods $(\tau=400$, or $t \approx 1 \mathrm{~ms})$.

\section{Mesh verification}

A mesh verification study was undertaken to establish confidence that the mesh resolution was sufficient to capture the surface pressure distribution and the unsteady features of the flow. Simulations were conducted on three meshes, with the properties shown in Table II.

Each mesh was structured, with hexahedral cells concentrated in the region of the jet orifice, as well as through the boundary layer and on the leading edge of the flat plate. The mesh spacing was increased linearly in all directions from these concentrated regions. Figure 3 shows the cell distribution for the coarse mesh, which has 38 cells across the jet diameter, and 34 cells within the experimentally measured boundary layer thickness $(\delta)$ upstream of the separation point, where $\delta=0.85 d_{j}[50]$.

The key parameters of interest in this study are the unsteady and time-averaged locations of the major flow structures, as these affect the surface pressure distribution and control force. Table II shows that the vertical distance 


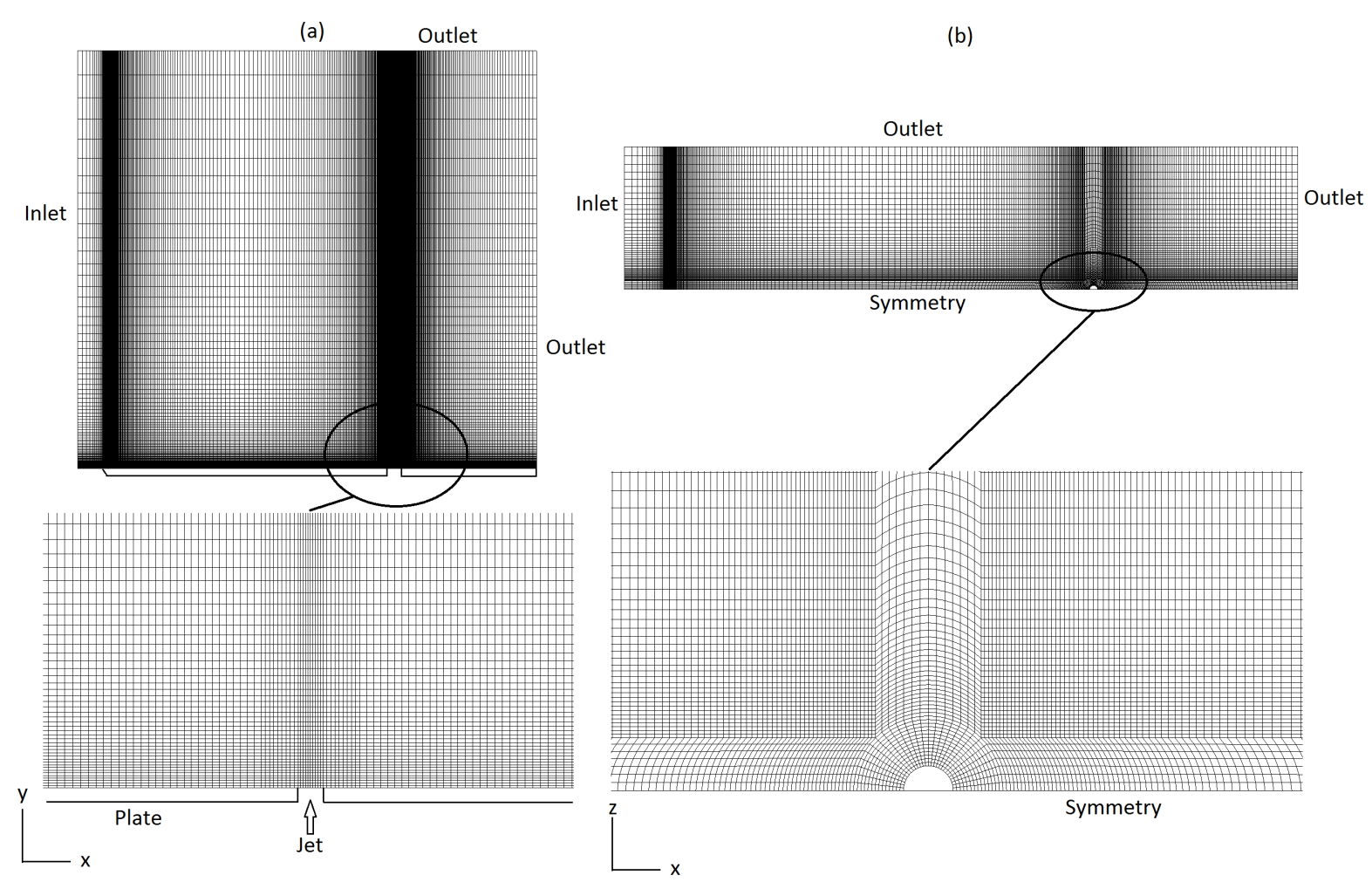

FIG. 3: Coarse mesh for jet in hypersonic crossflow simulations. (a) Side view; (b) Top view.

between the flat plate and the centre of the Mach disk, i.e. the Mach disk height $(h)$, is almost identical between the three meshes, with less than $2 \%$ variation. This confirms that the locations of the shock structures are independent of mesh resolution.

A commonly used method to establish whether mesh resolution is sufficient for LES is to compare the resolved turbulent kinetic energy (TKE) with the total TKE. It is generally recommended that if $80 \%-90 \%$ of the TKE is resolved, LES can be considered well-resolved [51, 52]. When using implicit LES, the unresolved, or sub-grid, TKE is not easily obtained. One possible approach is to directly apply an explicit sub-grid model to an instantaneous snapshot of the implicit LES solution. One frequently used explicit sub-grid model is Smagorinksy-Lilly, where sub-grid TKE $\left(k_{S G S}\right)$ is expressed as [52]:

$$
k_{S G S}=\nu_{t}^{2} /(c \Delta)^{2}
$$

where $c=0.094$ and $\Delta$ denotes the filter width. OpenFOAM uses a "top hat" filter, where $\Delta$ corresponds to the cell size. $\nu_{t}$ is the turbulent viscosity, which can be calculated from the rate of strain tensor as follows:

$$
\nu_{t}=(c \Delta)^{2} \sqrt{2 \times S_{i j} \times S_{i j}}
$$

where the rate of strain tensor is defined as:

$$
S_{i j}=\frac{1}{2}\left(\frac{\partial u_{i}}{\partial x_{j}}+\frac{\partial u_{j}}{\partial x_{i}}\right)
$$

This method allows a direct estimate of the sub-grid TKE, which can be added to the resolved TKE to compute the total TKE. The resolved TKE $\left(k_{G S}\right)$ is simply:

$$
k_{G S}=\frac{1}{2}\left(u_{1}^{\prime 2}+u_{2}^{\prime 2}+u_{3}^{\prime 2}\right)
$$




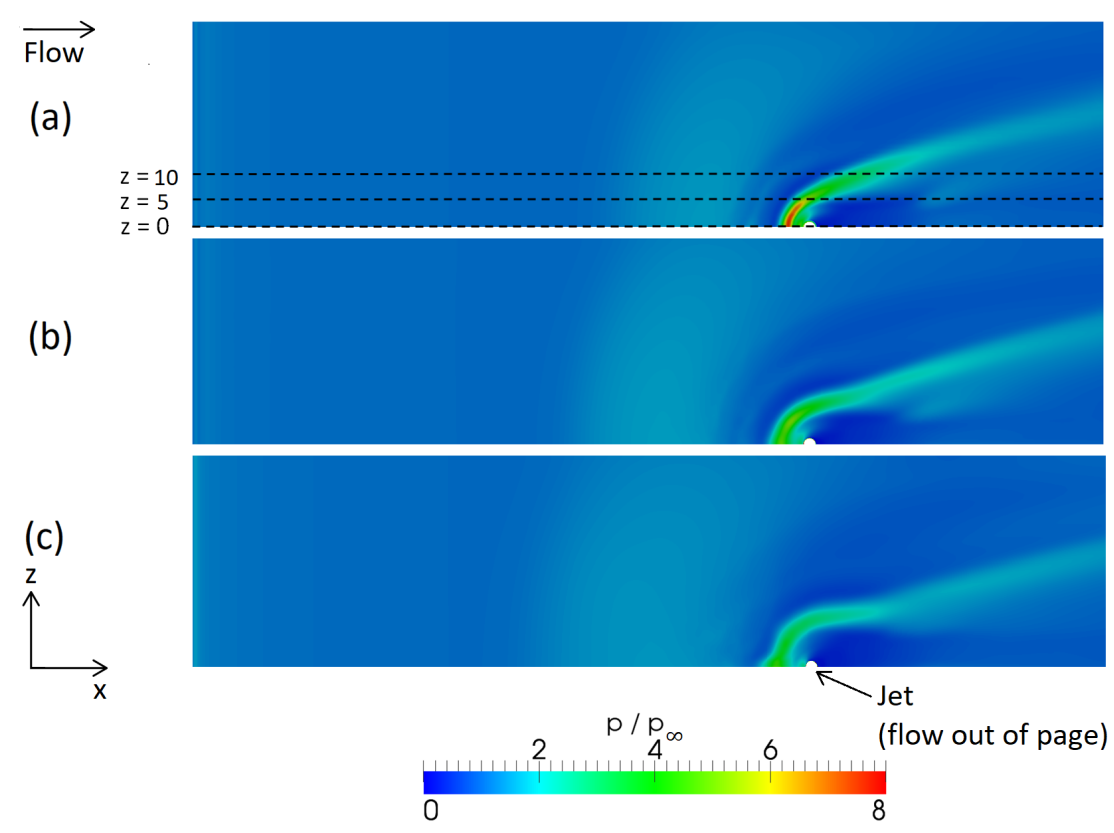

FIG. 4: Comparison of time-averaged surface pressure distribution for: (a) coarse mesh (b) intermediate mesh, and (c) fine mesh.

where $u_{i}^{\prime}=u_{i}-\overline{u_{i}}$, and $\overline{u_{i}}$ is the time-averaged velocity.

From this analysis, the ratio of resolved to total TKE integrated over the entire domain was found to be greater than $99 \%$ for the coarse mesh. Kinetic energy resolution is high because the Reynolds number is relatively low, thus allowing resolution of most of the turbulent kinetic energy. In addition, with a laminar in-flow, turbulence is limited to a small region of the domain where the jet-in-crossflow interaction occurs. The ratio of sub-grid to resolved TKE is very small (i.e. less than $0.1 \%$ ) at all locations throughout the domain and is maximum at the leading-edge shock, far from the jet interaction region where the mesh is concentrated. Performing the same calculation for intermediate and fine meshes shows the unresolved TKE is negligible throughout the domain.

Figure 4 shows the time-averaged pressure distribution on the flat plate for the three meshes. The coarse mesh solution predicts a higher peak pressure in the re-circulation region upstream of the orifice compared with the intermediate and fine meshes. The fine mesh result shows some distortion in the bow shock, due to the improved resolution of vortical structures. With this exception, the fine mesh closely matches the intermediate mesh.

Figure 5 provides a quantitative comparison of the time-averaged surface pressure at three span-wise locations marked on Figure 4 (a), and confirms that the solution is similar on the fine and intermediate mesh. The pressure peak on the jet centre-line is within $5 \%$ for the intermediate and fine mesh, but is $50 \%$ higher on the coarse mesh. The primary difference between the intermediate and fine mesh solution is the boundary layer separation point, which moves upstream from the jet as mesh resolution is increased. It has been shown previously that prediction of boundary layer separation is a difficult task [53], and this remains true with the current MILES methodology. With reference to the intermediate mesh, the separation point is $9 \%$ further downstream on the coarse mesh, and $10 \%$ further upstream on the fine mesh. The temporal variation in separation location increases from $\pm 3 \%$ to $\pm 5 \%$ to $\pm 10 \%$ as mesh resolution increases. Therefore, the time-averaged estimate on the intermediate mesh is within the simulated temporal variations on the fine mesh. In addition, time-averaged Mach disk height predictions are consistent between meshes. Therefore, it is concluded that the intermediate and fine meshes predict consistent time-averaged pressure distributions and shock locations.

To evaluate the performance of MILES in comparison to conventional LES, Figure 5 includes results using a Smagorinksy LES sub-grid model [52] on the intermediate mesh. The conventional LES results are very similar to the MILES. The peak pressure on the jet centre-line is between the coarse and intermediate mesh MILES result, $20 \%$ higher than the intermediate mesh MILES. The boundary layer separation point is within $5 \%$ of the MILES result on the same mesh. This shows that the MILES methodology has comparable performance to conventional LES when predicting surface pressure; however, the explicit LES model adds additional dissipation.

Further comparison can be made by considering the velocity profiles downstream of the jet outlet. The result is similar on all three meshes, and is also similar to a conventional LES solution. The coarse mesh result slightly 
(a)

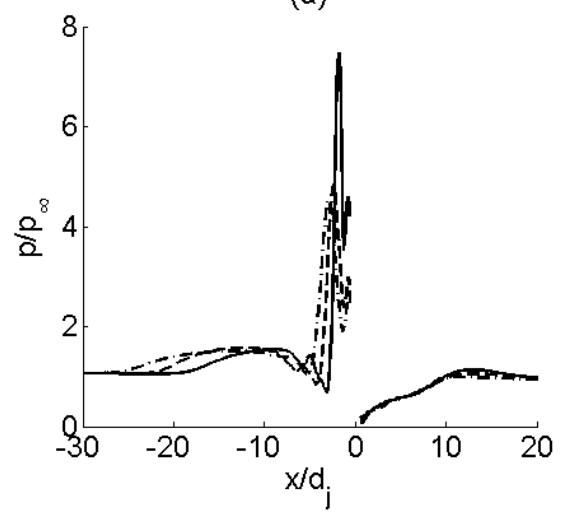

(b)

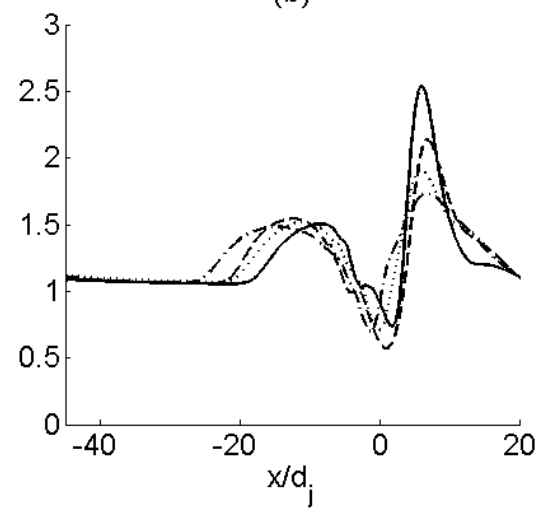

(c)

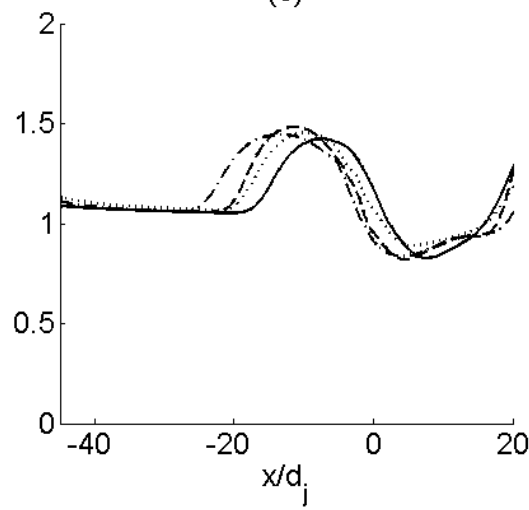

FIG. 5: Comparison of time-averaged pressure on the plate between meshes for: (a) $z / d_{j}=0$, (b) $z / d_{j}=5$, and (c) $z / d_{j}=10$. Solid line $=$ coarse mesh, dash line $=$ intermediate mesh, dot-dash line $=$ fine mesh, dotted line $=$ Smagorinsky sub-grid model (intermediate mesh).

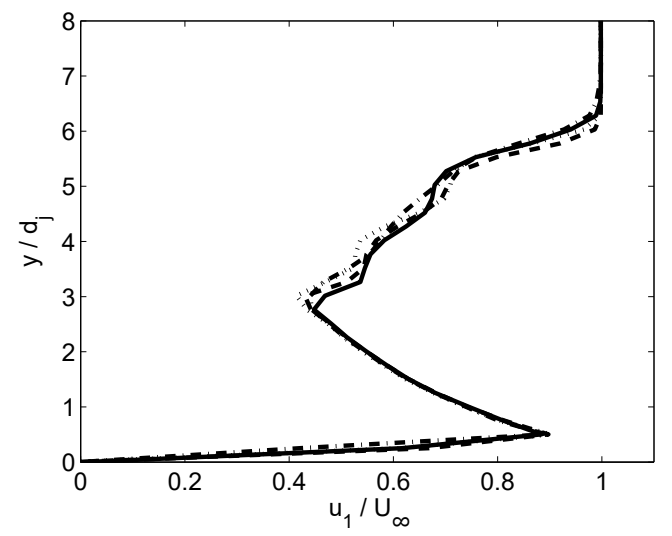

FIG. 6: Velocity profiles, measured on the jet centre-line, one jet diameter downstream of the orifice $\left(x=d_{j}\right)$. Solid line $=$ coarse mesh, dash line $=$ intermediate mesh, dot-dash line $=$ fine mesh, dotted line $=$ Smagorinsky sub-grid model (intermediate mesh).

under-predicts the maximum velocity deficit, while all three meshes predict the a similar vertical extent of the jet and boundary layer thickness.

Temporal variations in pressure were measured at a number of locations in the flow. The mean and RMS values on the plate, $5 d_{j}$ downstream of the jet outlet, are provided in Figure 7 . The mean pressure coefficient on each mesh is consistent within 5\%, while the temporal variations increase as mesh resolution increases and more turbulent structures are resolved near the wall. A Richardson Extrapolation [54] was performed on the RMS pressure variations. This gave an order of convergence of 1.4 , and convergence errors of $21 \%, 41 \%$, and $73 \%$ for the fine, intermediate, and coarse meshes respectively. This confirms that the temporal variations are being progressively more resolved as mesh resolution increases.

Further insight was gained by investigating the spectra of the pressure signal at the same location, $5 d_{j}$ downstream of the jet outlet. The spectra are provided in Figure 8. Consistent with the results shown above, the spectra are similar at low frequency, and the finer mesh resolves more energy at higher frequency.

Results were also compared between the current computational domain and a full three-dimensional domain on the intermediate mesh, with the plane of symmetry removed from the jet centre-line. The flow structures were similar for both simulations. For example, the time-averaged pressure distribution is shown in Figure 9. Similar results were observed for instantaneous pressure and three-dimensional flow structures (not shown for brevity), confirming that the use of a symmetry plane does not significantly influence the results for this flow.

These results provide confidence that the MILES resolves nearly all the TKE, and progressively resolves more turbulent structures with increased mesh resolution. The demonstrated similarity in shock structure and pressure distribution between the intermediate and fine mesh indicates that the intermediate mesh is sufficient for this study. 


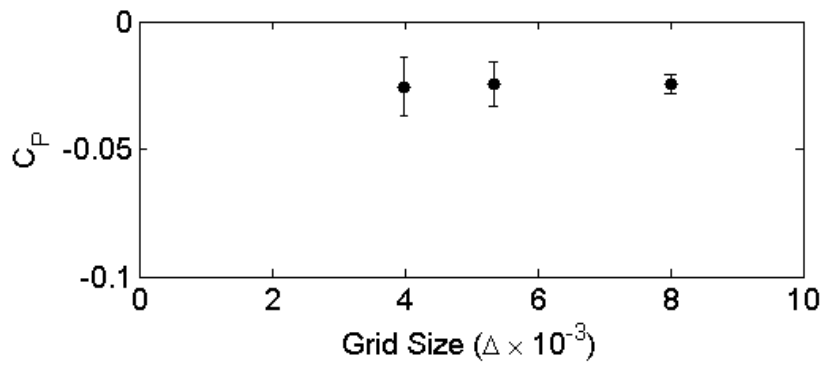

FIG. 7: Mean and RMS pressure coefficient on the plate for each mesh, on the jet centre-line, 5 jet diameters downstream of the jet outlet.

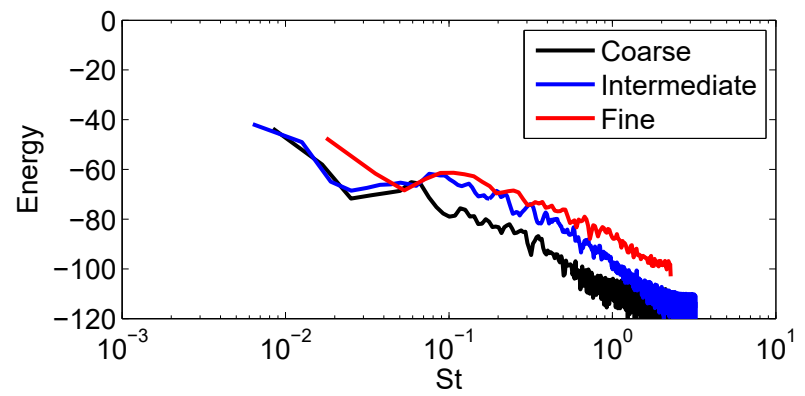

FIG. 8: Spectra for pressure on the plate on the jet centre-line, 5 jet diameters downstream of the jet outlet.

\section{Validation}

Parameters considered for validation were time-averaged shock structure and surface pressure, and the dimensions associated with primary flow features, specifically mean boundary layer thickness $(\delta)$, Mach disk height $(h)$ and separation location $\left(x_{s e p}\right)$. These parameters were quantitatively compared to experimental results [48].

Shock structures were compared using time-averaged density gradient contours from the simulation, compared with the experimental Schlieren images in Figure 10. All major shock structures in the flow were captured by the simulation, including the leading edge shock, separation shock, bow shock, and Mach disk. A re-compression shock was not directly observed in the plane of symmetry by either the experimental or numerical Schlieren images. Overall, the simulated flow structure is in good agreement with the experiment, and the time-averaged flow features are reasonably

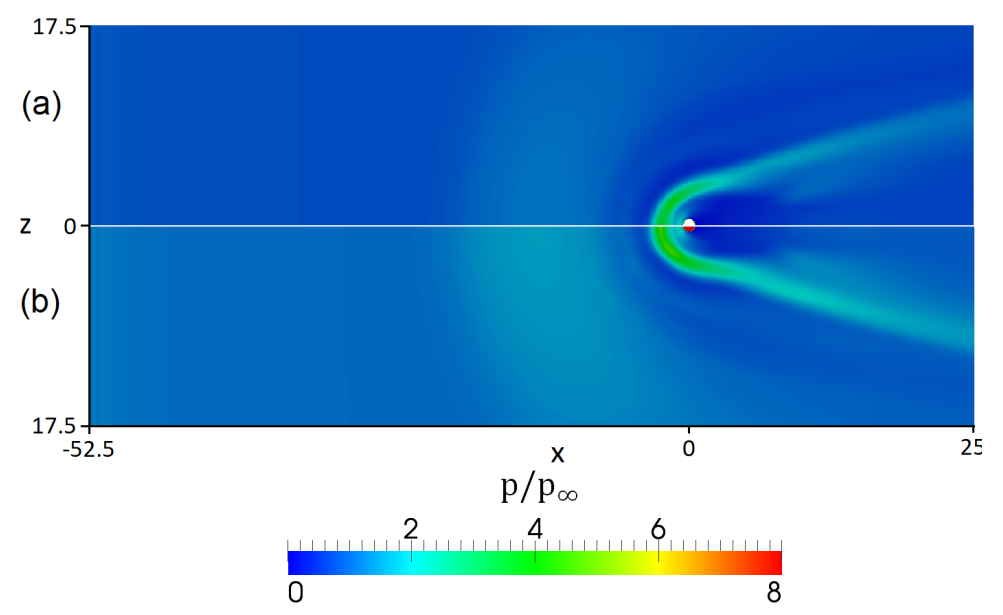

FIG. 9: Comparison of time-averaged surface pressure distribution for: (a) current domain, and (b) full three-dimensional domain. 
TABLE III: Validation results.

\begin{tabular}{cccc}
\hline & $\delta / d_{j}$ & $h / d_{j}$ & $x_{\text {sep }} / d_{j}$ \\
\hline Simulation & 0.46 & 3.4 & 25 \\
Theory & 0.6 & 4.3 & - \\
Experiment & 0.85 & 4.1 & 22 \\
\hline
\end{tabular}

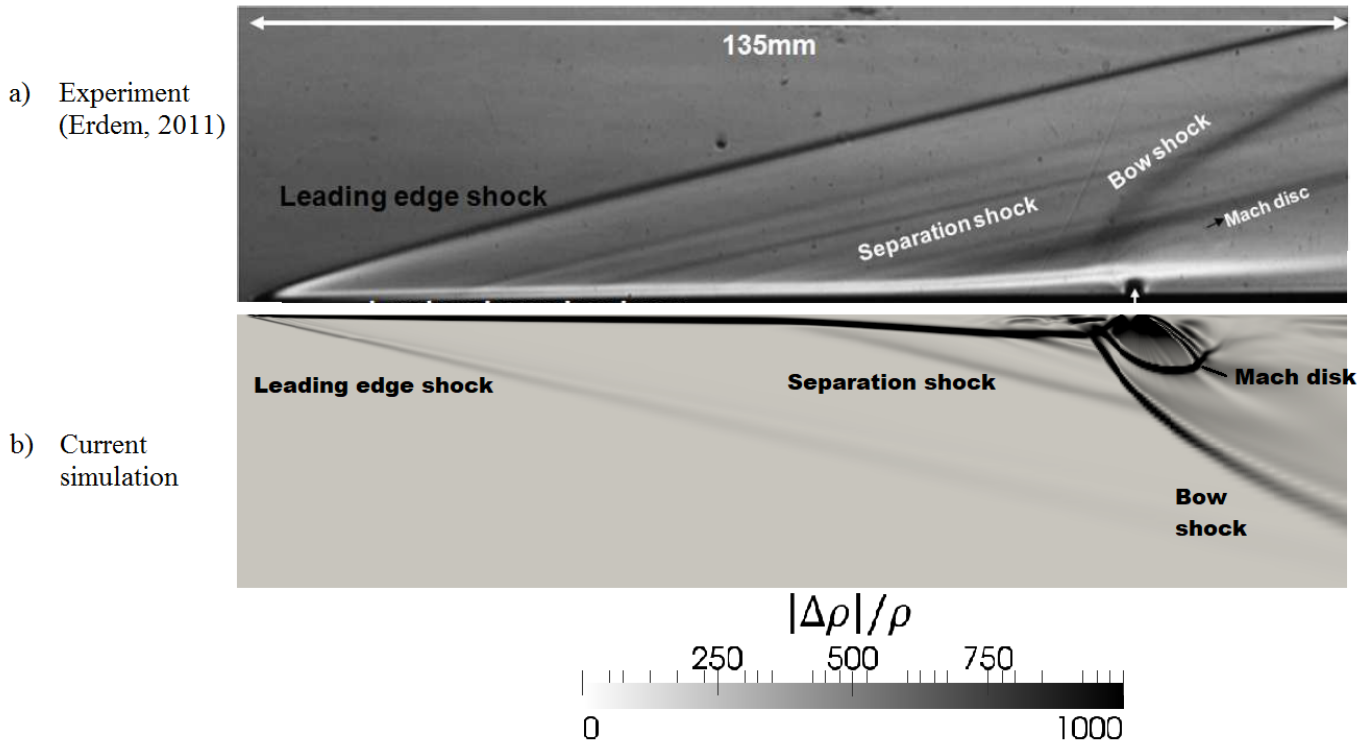

FIG. 10: Comparison of (a) experimental Schlieren visualisation [48], and (b) time-averaged density gradient contours in the plane of symmetry.

well captured. The main difference is the height of the Mach disk, which is $20 \%$ higher in the experiment. This is caused by the difference in boundary layer thickness, which is caused by the different temperatures of the flat plate.

In the experiment, boundary layer thickness was measured on the jet centre-line, $x / d_{j}=22$ upstream of the jet. These results, and the corresponding simulation results are provided in Table III, along with the analytical estimate [55] based on a reference temperature method. The discrepancy between simulation and the analytical estimate is due to the temperature of the plate. In the simulation, the plate was initialised at the free-stream temperature $(62.5 \mathrm{~K})$, while the analytical adiabatic wall temperature is $321 \mathrm{~K}$ [55]. An additional simulation was conducted on the coarse mesh, with the plate temperature fixed at $321 \mathrm{~K}$. The error between simulated and analytical values was reduced from $23 \%$ to $11 \%$. The remaining $11 \%$ error is attributed to mesh resolution; the simulated boundary layer thickness is within 2 cells of the analytical value. The experimental wall temperature was not published, but is expected to be initially at room temperature $(\approx 300 \mathrm{~K})$, close to the adiabatic wall temperature. The experimental boundary layer thickness exceeds the analytical estimate by $42 \%$, and this discrepancy is attributed to leading edge bluntness, and surface roughness.

The thicker boundary layer in the experiment allows the jet to penetrate further before it is deflected by the crossflow. Therefore, the Mach disk is expected to form further from a plate with a thicker boundary layer. This corresponds to the observed behaviour (see Table III). The influence of boundary layer thickness on Mach disk height was confirmed by running an additional simulation on the coarse mesh with an extended plate length, such that the boundary layer thickness was matched to the experiment at the measurement location. This reduced the discrepancy between experimental and simulated time-averaged Mach disk height from 20\% to 6.8\%. The remaining discrepancy is attributed to mesh resolution and unsteadiness in the Mach disk. Further confirmation is provided in Figure 11 (a), where Mach disk height is normalised by boundary layer thickness and compared to previously published data. The current simulation fits well within published experimental data. Figure 11 (b) compares the jet trajectory, normalised by jet diameter $\left(d_{j}\right)$ and jet-to-crossflow momentum ratio $(J)$ and shows that the current simulation again fits well with published data. The exception is Rogers [8], who observed increased penetration due to a thicker in-flow boundary layer.

Wall pressure distribution is compared between simulation and experiment in Figure 12. In the experiment, wall 
(a)

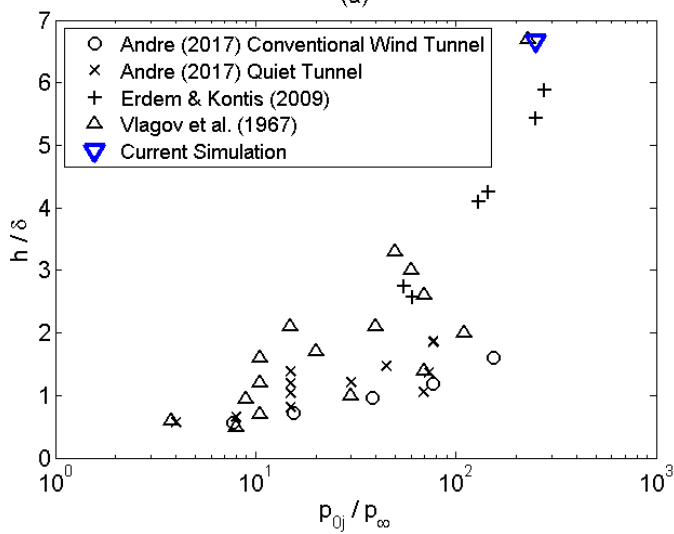

(b)

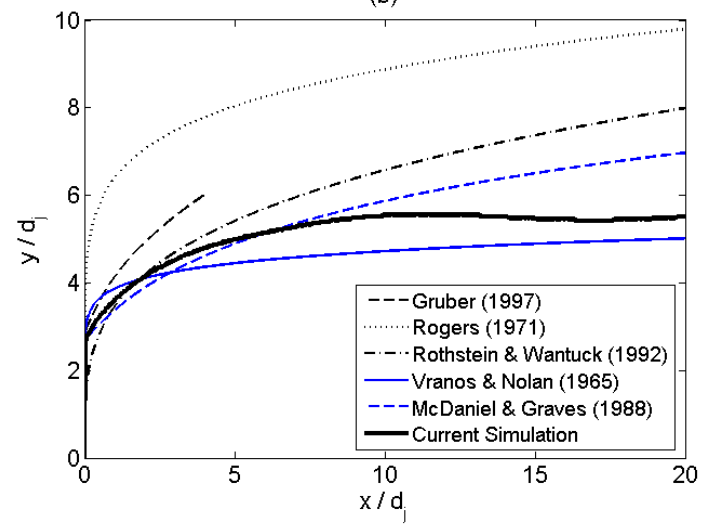

FIG. 11: (a) Mach disk height as a function of pressure ratio, comparing the current simulation to data compiled by Andre et al. [28, 56, 57], and (b) Jet trajectory comparing the current simulation to data compiled by Mahesh $[5,8,30,58-60]$.

(a)

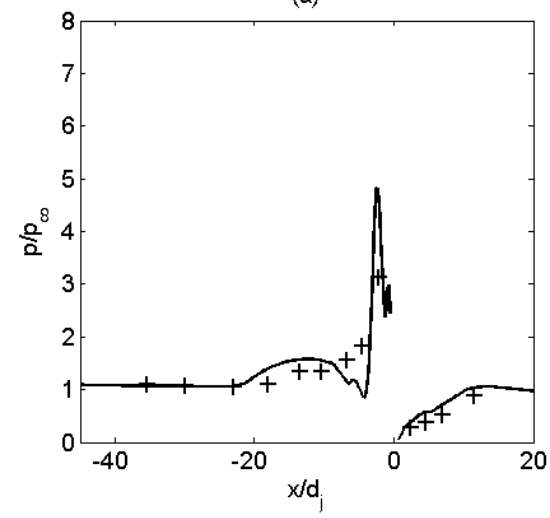

(b)

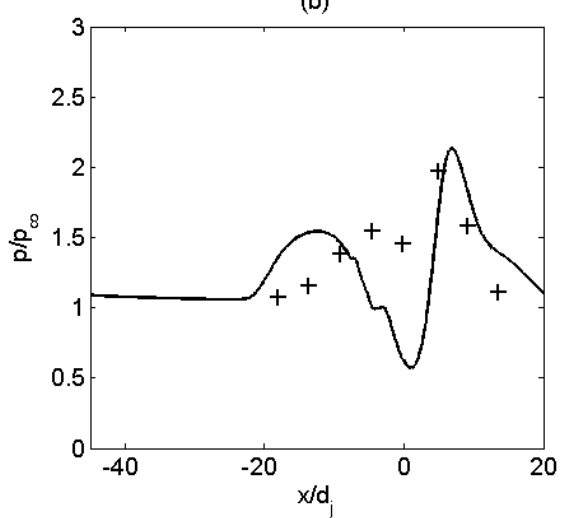

(c)

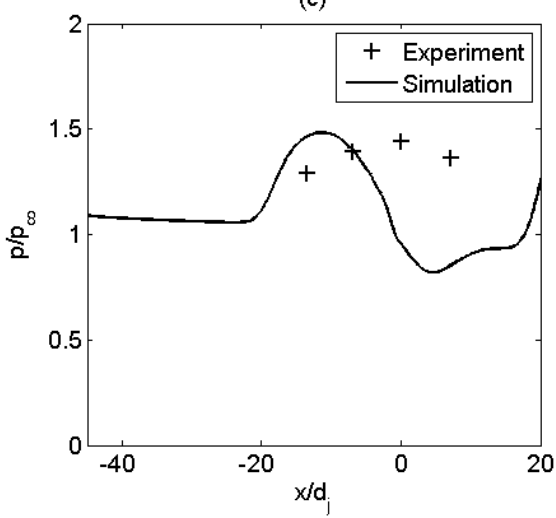

FIG. 12: Comparison of pressure on the plate between simulation and experiment for: $(\mathrm{a}) z / d_{j}=0,(\mathrm{~b}) z / d_{j}=4.8$, and $(\mathrm{c}) z / d_{j}=9.1 .+=$ Experiment; Solid line $=$ Simulation.

pressure was measured by pressure taps on the plate along the jet centre-line $\left(z / d_{j}=0\right)$ and at lateral distances of $z / d_{j}=4.8$ and $z / d_{j}=9.1$ (approximate measurement locations are shown in Figure 4 (a)). Qualitatively, the simulation predicts pressure well along the jet centre-line. The separation location shows a small discrepancy, while the high pressure region upstream of the jet outlet and the low pressure region downstream of the jet are well predicted.

At $z / d_{j}=4.8$, the overall behaviour is predicted correctly. The pressure increases at the point of boundary layer separation, which is slightly further upstream in the simulation, then decreases before a large increase at the bow shock location. Behind the bow shock, the pressure recovers to the free-stream value. The simulated pressure drop upstream of the bow shock (at around $x=0$ ) is not captured in the experiment, and is slightly further upstream in the simulation both at the centre-line, and off-centre, due to the separation point being further upstream.

At $z / d_{j}=9.1$, the comparison appears poor, as the experimental data only captures the upstream re-circulation region, which is further upstream in the simulation.

Overall, the agreement with experimental data is good, once the different boundary layer separation point is taken into account.

The final parameter considered is the length of the separation region $\left(x_{s e p}\right)$, which was reasonably well predicted, within $15 \%$ of the experimental value. The discrepancy between simulation and experiment is most likely due to differences in boundary layer thickness, plate temperature, surface roughness, and leading edge bluntness. As noted previously, numerical and experimental measurements for separation position rarely agree, and there are significant temporal variations in the simulated separation point, so this result is reasonable.

Overall, the simulation correctly predicts all major features of the flow observed in the experiment. Differences between simulation and experiment are attributable to differences in plate temperature and physical properties. Simulation results also closely match analytical estimates and previously published data. 


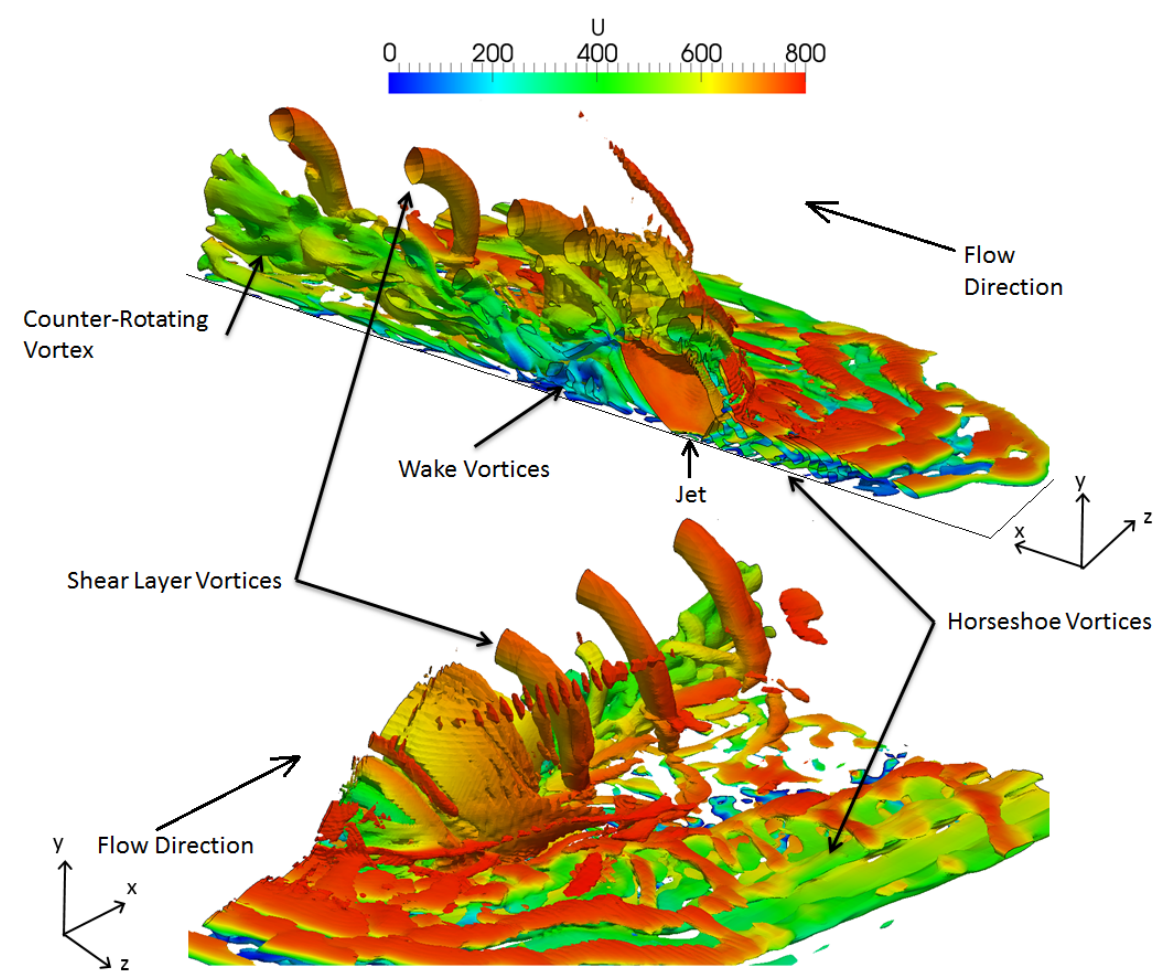

FIG. 13: Isometric contours of Q-criterion, for $Q=10^{10}$, coloured by velocity magnitude, showing instantaneous flow structure.

\section{RESULTS AND DISCUSSION}

\section{A. Overall flow structure}

The instantaneous structure of the flow is shown in Figure 13. Horseshoe vortices, shear-layer vortices (or hairpin vortices), wake vortices, and a stream-wise counter-rotating vortex are identified. Horseshoe vortices form in the re-circulation region, upstream of the jet outlet, and bend around the jet forming the characteristic horseshoe shape. Here, there are several vortices rather than a single, well-defined horseshoe vortex. Hairpin type shear-layer vortices can be seen travelling downstream after being periodically shed from the shear-layer near the upstream barrel shock. The counter-rotating vortex also becomes clear in the far-field, while wake vortices are irregularly shed from the vicinity of the leeward barrel shock.

Figure 14 shows contours of the Q-criterion in the plane of symmetry, on the plate, and in a span-wise plane perpendicular to the crossflow $20 d_{j}$ downstream of the jet orifice. Figure 14 compares the vortex structures present in the instantaneous and time-averaged flow-fields. In the instantaneous flow-field, a complex system of horseshoe vortices, and distinct shear-layer vortices can be seen in plane of symmetry. The span-wise plane shows the individual vortices that comprise the single stream-wise counter-rotating vortex observed in the time-averaged flow. The spanwise plane also shows the trajectory of the horseshoe vortices. Vortices that form close to the jet merge back into the plane of symmetry, while vortices formed further upstream persist far downstream along the plate.

The shock structure can also be clearly visualised in Figure 14, and is similar to the well documented jet-insupersonic-crossflow shock structure shown in Figure 1, and to the experimentally measured shock structure in Figure 10.

\section{B. Surface pressure distribution}

Figure 15 (a) shows the time-averaged pressure on the plate. Along the jet centre-line $(z=0)$, there is a clear increase in pressure upstream of the jet outlet, from the point of boundary layer separation, at approximately $-25 \lesssim x / d_{j} \lesssim-8$. Nearer the jet, the pressure drops, before a large peak in front of the jet outlet, corresponding to the bow shock. 
a)

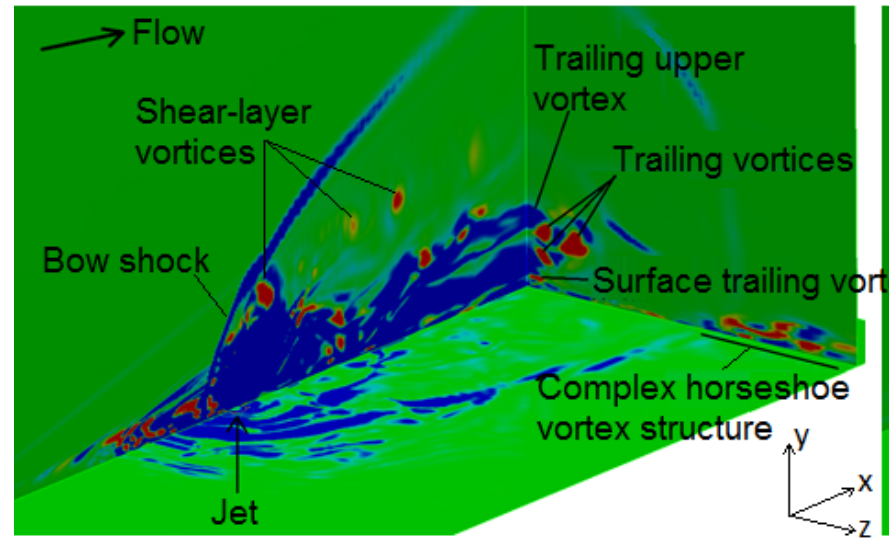

b)

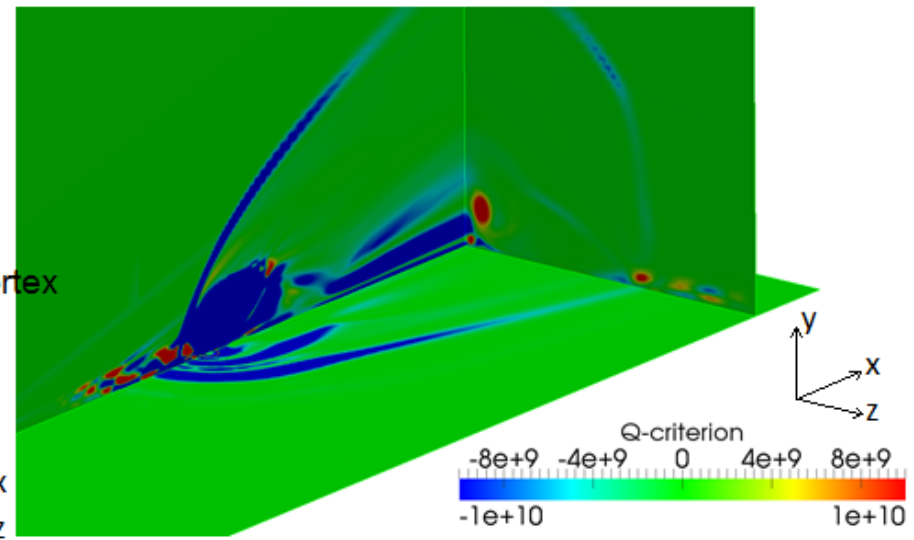

FIG. 14: Contours of Q-criterion, with cutting planes in the plane of symmetry, on the plate, and perpendicular to the freestream, 20 jet diameters down-stream of the jet outlet, showing (a) Instantaneous and (b) Time-averaged flow structure.
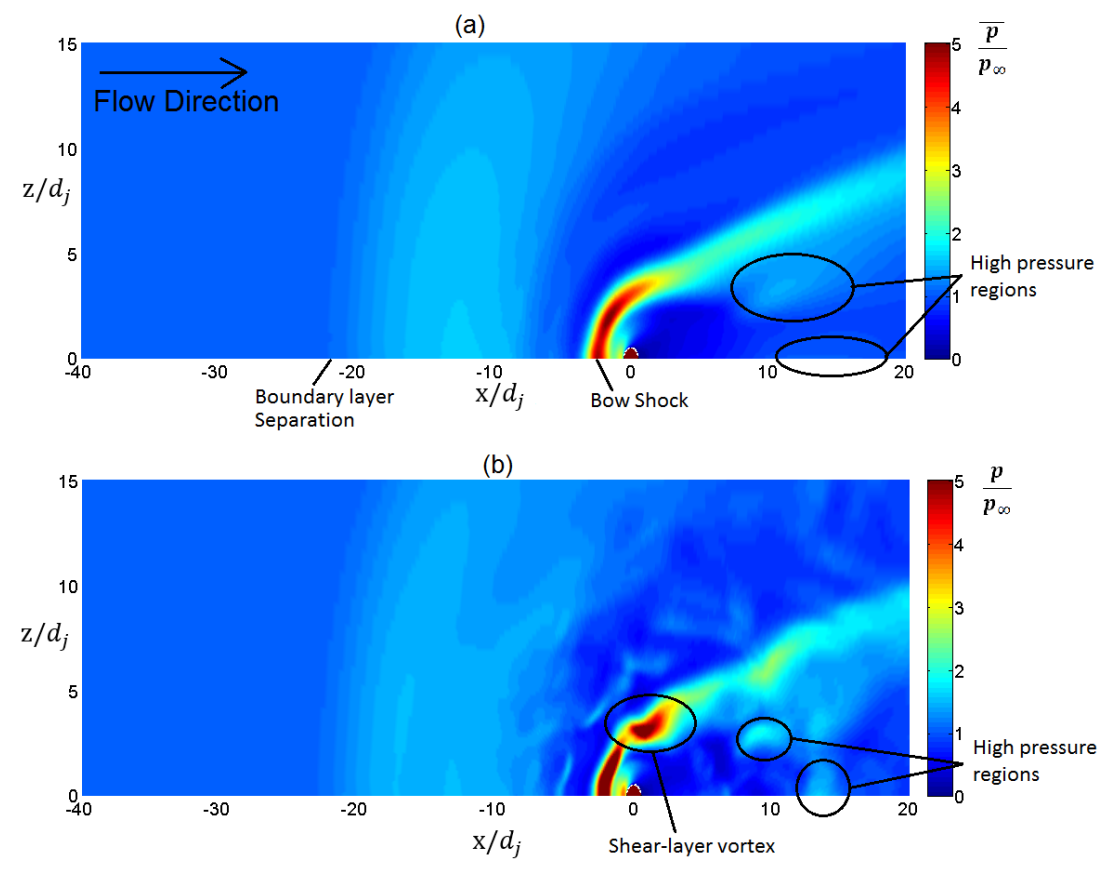

FIG. 15: (a) Time-averaged, and (b) Instantaneous pressure contours on the plate.

A re-circulation region forms in this area immediately upstream of the jet outlet. The pressure downstream of the jet is reduced below the free-stream value, as the free-stream flow is obstructed by the jet. Further downstream, at $x / d_{j}>10$, the pressure recovers. The pressure in this region exceeds the free-stream value, as the free-stream flow that moved laterally around the bow shock flows back into the wake region. Moving away from the jet centre-line, the overall behaviour remains the same. The boundary layer separation point, and the jet bow shock curve around the jet outlet, while peak pressure is decreased due to weakening of the bow shock. As indicated on Figure 15 (a), there are isolated, localised regions of higher pressure behind the bow shock, both away from the jet centre-line and on the centre-line.

Figure 15 (b) shows the instantaneous pressure on the plate. This allows a more detailed investigation of the individual structures within the flow that influence the pressure distribution. The overall features of the time-averaged pressure field are maintained, but individual flow structures are resolved. Figure 15 (b) shows a strong high pressure region lateral to the jet that is not present in the time-averaged data, corresponding to the location of a shear-layer 


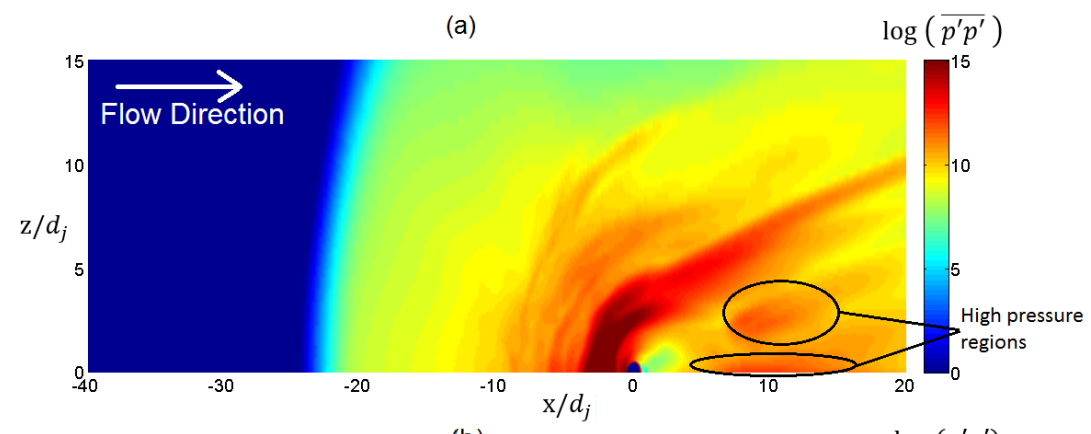

(b)

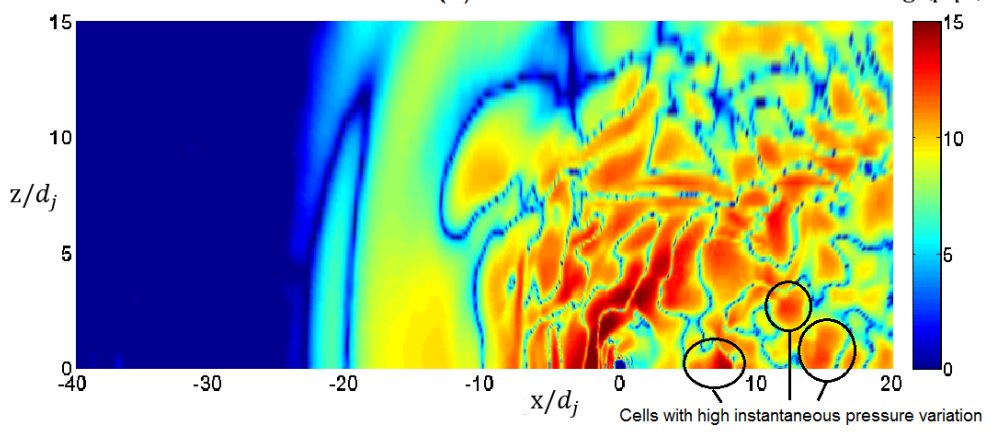

FIG. 16: Variation in pressure on the plate, plotted on a logarithmic scale. (a) Mean-squared $\left(\overline{p^{\prime} p^{\prime}}\right)$, and (b) Instantaneous $\left(p^{\prime} p^{\prime}\right)$ pressure variation.

vortex. This high pressure region will convect downstream. The instantaneous image also shows small, high pressure regions which give rise to the localised high pressure regions in the time-averaged data.

Temporal pressure variations are shown in Figure 16. Figure 16 (a) shows the square of the time-averaged pressure variation (i.e. the mean-squared variation), $\overline{p^{\prime} p^{\prime}}$, where $p^{\prime}=p-\bar{p}$, and $\bar{p}$ is the time-averaged pressure. Figure 16 (b) shows the instantaneous variation in pressure, squared (i.e. $p^{\prime} p^{\prime}$ ). The instantaneous data reveals a cellular structure, in which individual cells correspond to the location of individual flow structures. The localised regions of high pressure highlighted in Figure 15 (a) are clearly identified as regions which also have high temporal variations in pressure. For example, large variations can be observed in the bow shock region, where the pressure is high. The other region with large pressure variations is in the re-circulation region upstream of the bow shock. This is caused by the changing structure of the horseshoe vortices. Lateral to the jet, the region of large fluctuations at the bow shock location splits, two separate regions can be seen in both the time-averaged and the instantaneous data. This is caused by the bow shock deformation that corresponds to shear-layer vortex shedding.

\section{Horseshoe vortices}

The cellular structure observed within the upstream separated flow region in Figure 16 (b) is driven by the horseshoe vortex structure. The boundary layer separates upstream of the jet, resulting in an adverse pressure gradient and a large region of re-circulating flow. A complex system of horseshoe vortices develops within the re-circulation region. This region of separated flow extends in the stream-wise and span-wise directions.

Figure 17 shows instantaneous span-wise vorticity in the symmetry plane, upstream of the jet exit, overlaid with streamlines. Six vortices are observed, and the structure is more complex than both subsonic crossflow and supersonic crossflow with turbulent boundary layer cases. Vortex A is the quasi-steady re-circulation vortex immediately upstream of the jet, caused by the free-stream flow deflected downward from the stagnation point. Instead of a single counter-rotating horseshoe vortex, five additional discrete structures can be seen upstream of the re-circulation vortex. These are each labelled Vortex B, C, D, E and F in Figure 17.

Figure 18 shows the temporal evolution of the horseshoe vortex system over a period of $10 \tau$. Vortex A and D are quasi-steady, and are always present, while Vortex B and C, as well as Vortex E and F, are co-rotating pairs which periodically coalesce. The time period shown in Figure 18 approximately represents a single period of coalescence of the vortices, that is, Vortices $\mathrm{B}$ and $\mathrm{C}$, and Vortices $\mathrm{E}$ and $\mathrm{F}$ periodically coalesce with a frequency corresponding to a Strouhal number $S t_{\infty} \approx 0.1$, where $S t_{\infty}=f \times d_{j} / U_{\infty}$. In isolation, Vortex A and B form a counter-rotating 


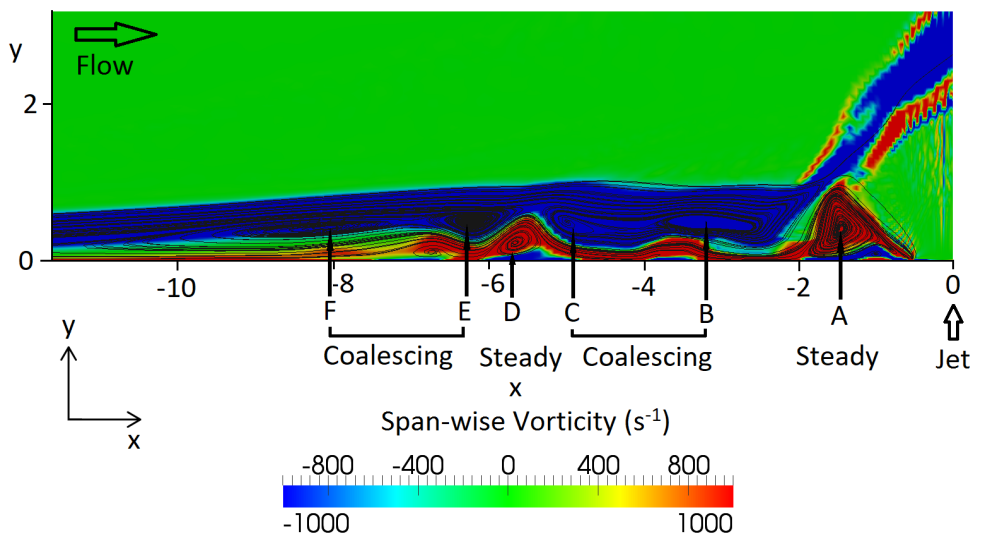

FIG. 17: Instantaneous span-wise vorticity in the symmetry plane, upstream of the jet outlet, overlaid with streamlines.
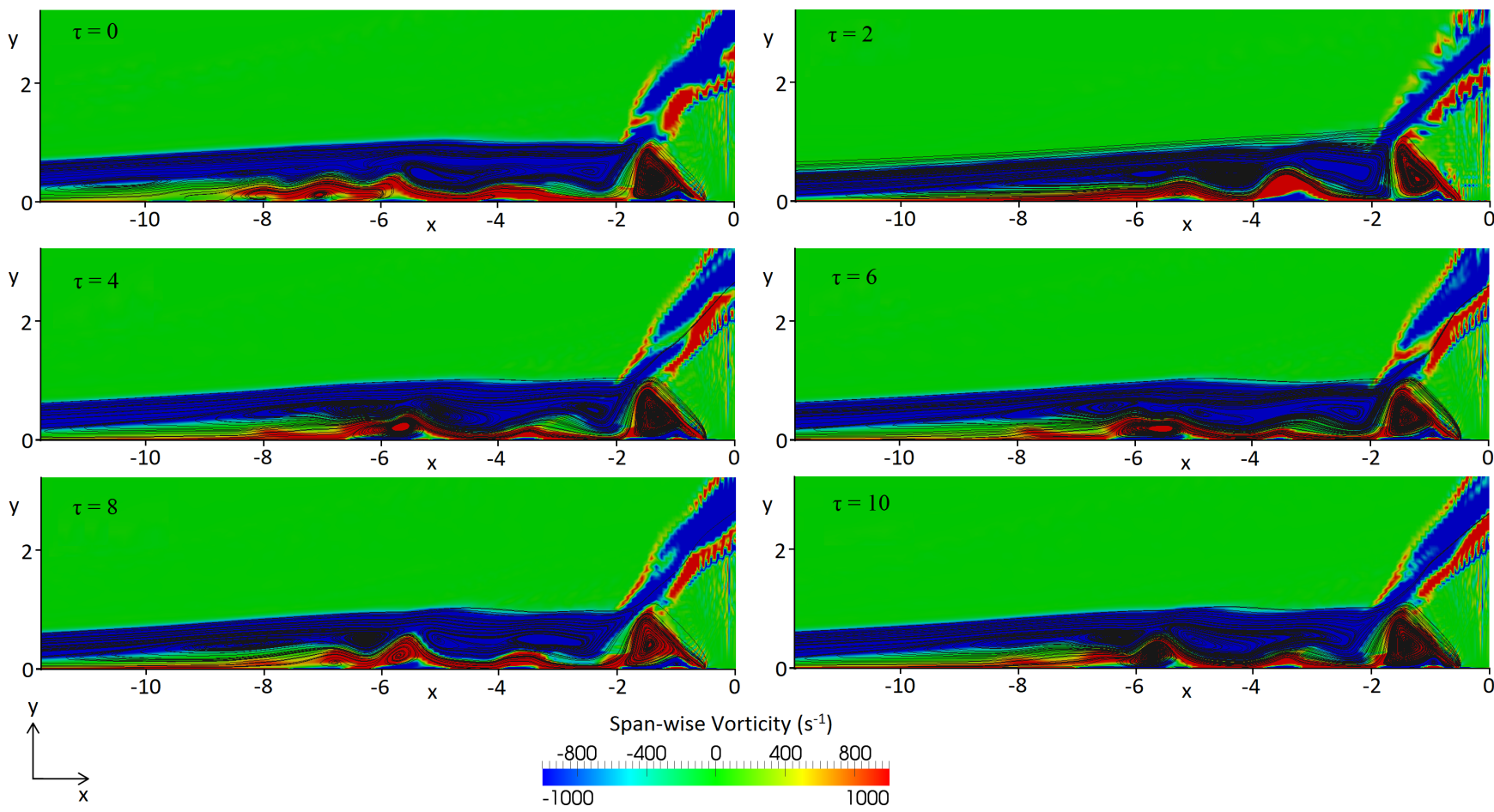

FIG. 18: Instantaneous span-wise vorticity in the symmetry plane, overlaid with streamlines at successive times, showing temporal evolution of the horseshoe vortex system.

pair, consistent with the steady regime identified for subsonic flow [11]. As the separated region is much larger for a laminar boundary layer, additional vortices develop.

The three-dimensional nature of the horseshoe vortices is examined in Figure 19, which shows streamlines of timeaveraged fluid flow through the horseshoe vortex region, overlayed on contours of time-averaged vorticity magnitude. Figure 19 shows that the flow from the vortex closest to the jet outlet (Vortex A) wraps tightly around the jet orifice, and is then lifted from the plate and entrained into the longitudinal counter-rotating vortex pair far downstream of the jet outlet. This behaviour is in agreement with Viti et al.[14]. The flow from the upstream vortices (Vortices B, $\mathrm{C}, \mathrm{E}$ and $\mathrm{F}$ ) is distinctly different to Vortex A; the fluid travels around the jet along the flat plate, corresponding to the usual horseshoe vortex behaviour. Flow from Vortex D travels upstream and extends far into the span-wise direction. In the instantaneous flow, some of the fluid from the horseshoe vortex system is carried upstream within the boundary layer, back to the point of boundary layer separation. The flow from the upstream vortices remains between the bow shock and separation shock, so does not influence the downstream development of the jet, while flow 
(a)

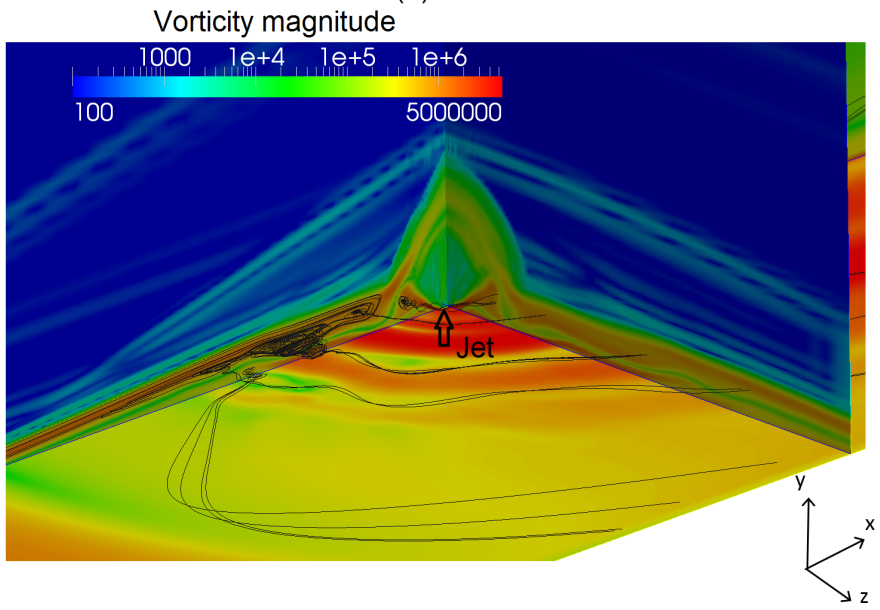

(b)

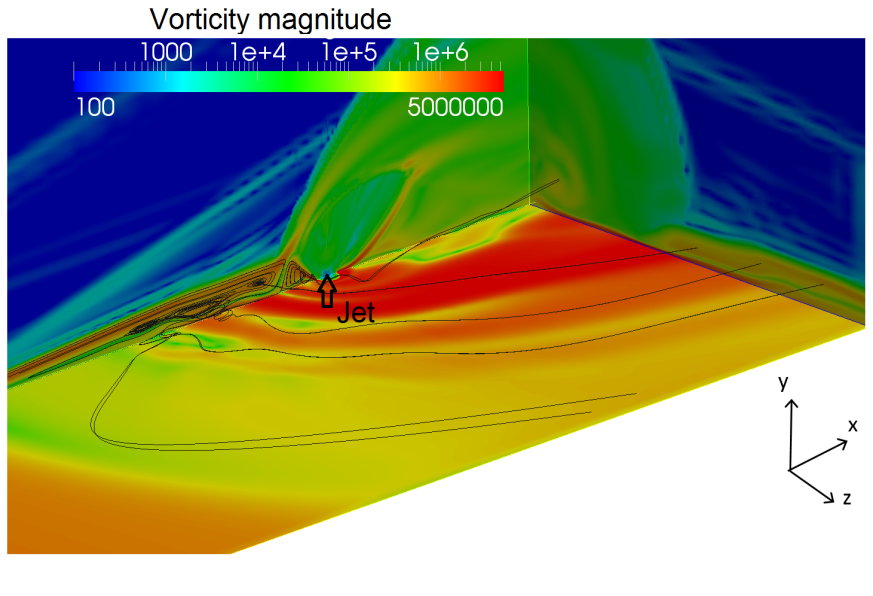

FIG. 19: Streamlines from horseshoe vortices overlaid on contours of time-averaged vorticity magnitude, with span-wise cutting planes at: (a) jet outlet, and (b) $10 d_{j}$ downstream.

(a)

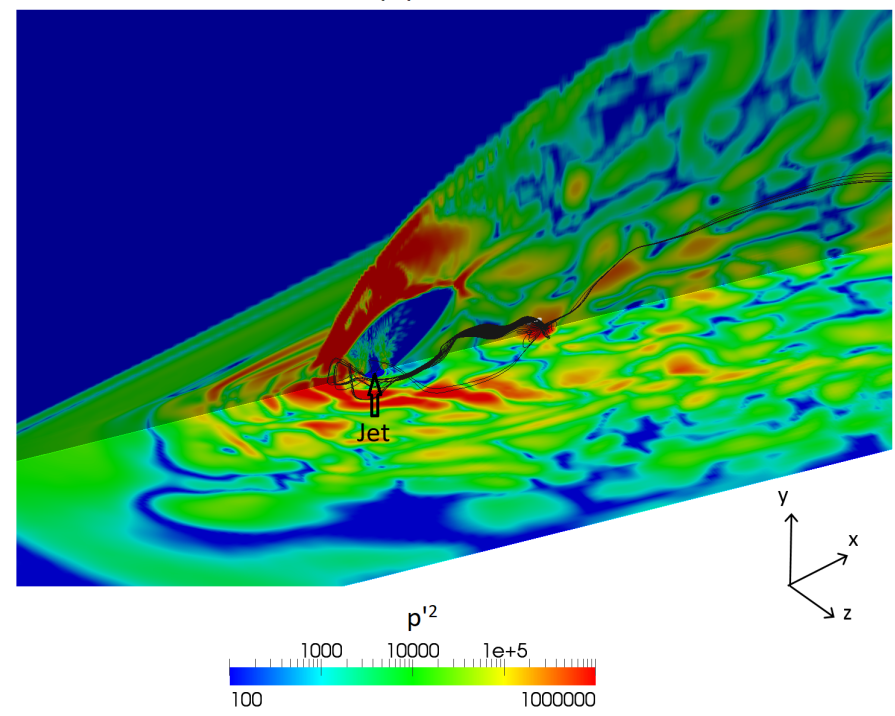

(b)

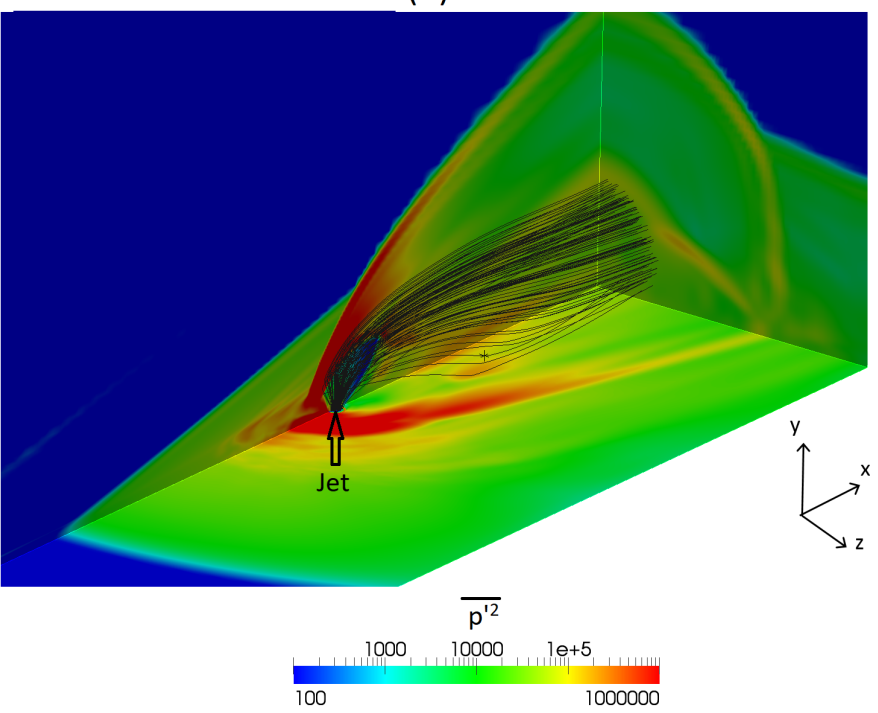

FIG. 20: Streamlines overlaid on (a) instantaneous pressure variation $\left(p^{\prime} p^{\prime}\right)$, and (b) mean-squared pressure variation $\left(\overline{p^{\prime} p^{\prime}}\right)$, showing flow into regions with high instantaneous pressure.

from Vortex A is entrained within the counter-rotating vortex, as mentioned.

The downward flow from the stagnation point into the re-circulation region (Vortex A) causes the high pressure region immediately upstream of the jet outlet. The orientation of Vortex A is in the opposite direction to the boundary layer vorticity. As a result, the flow is lifted from the plate as it travels downstream around the jet outlet, so the pressure on the plate in the region between the bow shock and the jet outlet reduces in the span-wise direction. As the flow from Vortex A moves around the jet outlet, it moves upward and laterally, until it emerges from the boundary layer and is forced back downward by the free-stream flow accelerating around the jet outlet, and by the suction from the low pressure region behind the jet outlet. This converging flow causes the high pressure region in the plane of symmetry, downstream of the jet outlet, which is clear in the instantaneous flow. This behaviour is observed in Figure 20 (a), which shows instantaneous streamlines of flow from Vortex A overlaid on contours of pressure variation $\left(p^{\prime} p^{\prime}\right)$. This high pressure region is elongated in the time-averaged flow (Figure 16 (a)) as the convergence point changes with time. The pressure in this area is high, and has high temporal variability. Similar behaviour was also identified by Santiago and Dutton [35] for a jet in supersonic crossflow with a turbulent boundary layer.

The high pressure region away from the jet centre-line, identified in Figure 15, is caused by jet flow. The lateral 
expansion of the jet and the three-dimensional nature of the barrel shock results in jet flow being deflected downward. Figure 20 (b) shows this behaviour in the time-averaged flow. The downward flow in this region causes a slightly elevated mean pressure (see Figure 15 (a)), while the unsteady nature of the barrel shocks also makes this a region with large temporal pressure variations (see Figure 16 (a)).

\section{Shear-layer vortices}

The shear-layer vortices are important to the jet force, as the periodic shedding of these vortices induces high pressure regions on the plate, and affects the shape and location of the Mach disk and barrel shock structures. Shearlayer vortices form in the shear-layer between the upstream barrel shock and the bow shock. These vortices do not form immediately at the jet exit, but rather form above the stagnation point, approximately three jet diameters from the outlet. Once formed, the shear-layer vortices initially travel parallel to leading edge of the jet barrel shock, which is deformed by the presence of the shedding vortices. This behaviour corresponds to the observations of Santiago and Dutton, who observed periodic flattening of the barrel shocks on the windward side [35]. The physics here differs from previous studies, where turbulent in-flow boundary layers were considered [9, 16, 31, 35]. The laminar boundary layer in this study is thicker, which causes the shear-layer vortices to form much further from the jet outlet, while the hypersonic crossflow causes the vortices, once formed, to be quickly turned and convected downstream with a high velocity. The jet-to-crossflow momentum ratio here $(J=5.3)$ is also higher than previous studies, where $J<2$.

The convection velocity of the shear-layer vortices is constant in the region $3<x / d_{j}<20$, and has a magnitude of approximately $0.8 U_{\infty}$. At large downstream distances $\left(x / d_{j}>20\right)$, this convection velocity increases slightly, to approximately $0.85 U_{\infty}$, in agreement with previous work $[30,31]$.

As the shear-layer vortices convect downstream, they are stretched, compressed and rotated [31]. Here, the height and width of the vortices were measured in the plane of symmetry, using a threshold value of the Q-criterion of $10^{9}$. Contours of Q at successive times are shown in Figure 21. In this case, the shear-layer vortices all have a similar shape, with an average width approximately equal to the jet diameter, and an average height of approximately $1.5 \times d_{j}$.

In this study, the jet shear-layer vortex shedding frequency is $S t_{j}=f \times d_{j} / U_{j} \approx 0.4$, based on the jet outlet diameter and jet velocity. If the Strouhal number is based on the jet diameter and the free-stream velocity $\left(U_{\infty}\right)$, then $S t_{\infty}=f \times d_{j} / U_{\infty} \approx 0.17$, which closely corresponds to previous laminar boundary layer simulations at lower supersonic Mach number [36]. Ben-Yakar et al. [31] reported $S t_{j} \approx 1$ for a variety of jet injection velocities. However, Ben-Yakar et al. were able to resolve shear-layer vortices much closer to the jet outlet, within one jet diameter, due to the turbulent in-flow boundary layer and smaller re-circulation region. This highlights the influence of a laminar boundary layer on the physical mechanism for vortex shedding. The shear-layer vortices form further from the jet outlet, and shed at a lower frequency, but remain intact far downstream.

Kawai and Lele [16] observed shear-layer vortex shedding at $S t_{\infty} \approx 0.4-0.6$ with a turbulent in-flow boundary layer in a Mach 1.6 crossflow, with $J=1.7$. This frequency reduced to $S t_{\infty}=0.2$ with a laminar boundary layer of equal thickness [36]. Chai et al. reported shedding at $S t_{\infty} \approx 0.3$ at the same condition, with a turbulent boundary layer [25].

In a subsonic crossflow with a turbulent in-flow boundary layer, the oscillation frequency of the horseshoe vortices is much lower than the shedding frequency of the shear-layer vortices [11]. The current simulation supports this finding, with shear-layer shedding at $S t_{\infty}=0.17$ and horseshoe vortex coalescence at $S t_{\infty}=0.1$. In this case, the frequencies are much closer than those observed in subsonic flow, where horseshoe vortices oscillated with a frequency 40 times lower than the shear-layer vortex shedding frequency [11]. The frequency of shear-layer vortex shedding in supersonic crossflow is higher than subsonic crossflow, where values of $S t_{j}=0.2$ have been reported [13].

Further insight into the dominant frequencies can be gained by investigating spectra of the pressure and velocity signals. Both spanwise velocity and pressure spectra show peaks at the same frequency, due to strong coupling between the pressure fluctuations inside the re-circulation region, and the dynamics of the shocks accompanying vortex formation [36]. Figure 22 shows the pressure spectra at two locations on the flat plate: inside the upstream re-circulation region, and far downstream.

In the upstream re-circulation region, there is a series of small peaks. One peak corresponds to horseshoe vortex coalescence at $S t_{\infty}=0.1$, while other peaks are present at lower frequency. This behaviour differs from previous work [36], where a distinct peak was observed in the upstream re-circulation region with a laminar crossflow. However, the broad spectrum closely matches data from turbulent in-flow boundary layers [25, 36]. Downstream, there is a distinct peak corresponding to the shear-layer vortex shedding, at $S t_{\infty}=0.17$. This agrees with Chai et al [25], and confirms that shear-layer vortex shedding is a significant unsteady component of the flow in the downstream region. 

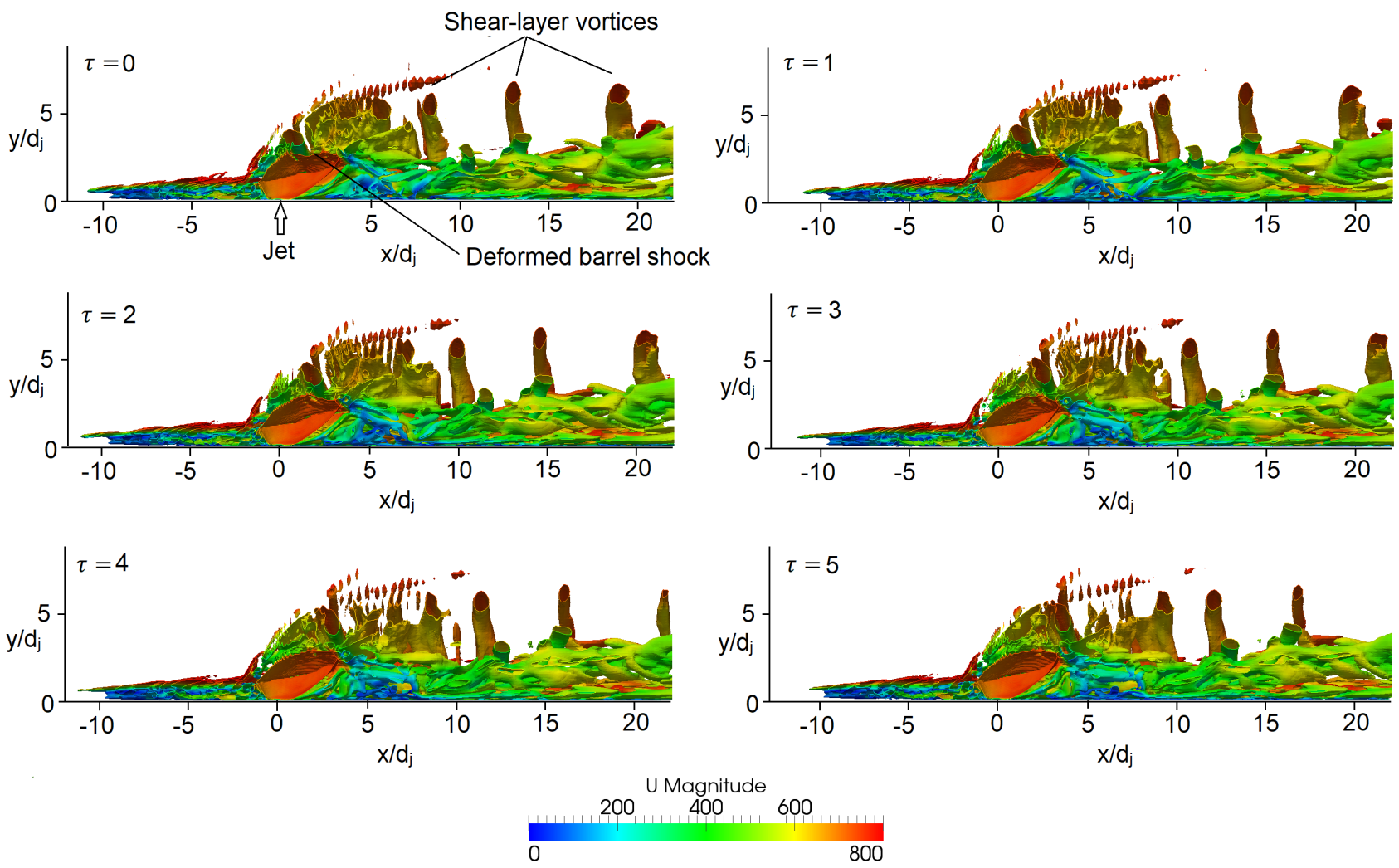

FIG. 21: Contours of $Q=10^{9}$ coloured by velocity magnitude in the plane of symmetry at successive times, showing shear-layer vortex shedding, and deformation of the barrel shock.

\section{E. Counter-rotating vortex}

Previous studies have identified the longitudinal counter-rotating vortex to be a significant contributor to the overall time-averaged pressure distribution, as this vortex structure dominates the interaction in the far-field [61]. The location and strength of this vortex plays a major role in determining the control force, as it is able to influence the pressure on the body over a large area.

The instantaneous and time-averaged counter-rotating vortex structure was shown in Figure 14. This stream-wise vortex is the dominant feature of the flow at large distances downstream of the jet outlet. The instantaneous structure consists of a series of trailing vortices, which rotate about a common axis, which form a single vortex in the timeaveraged flow, consistent with previous studies [14]. The extent of the time-averaged vortex $20 d_{j}$ downstream is approximately $3 d_{j} \times 3 d_{j}$ and is centred at $(y, z)=\left(2 d_{j}, 2 d_{j}\right)$. The single, coherent longitudinal vortex is not formed until $x / d_{j}>15$, which supports previous findings, that the single vortex structure in the far-field develops from several individual near-field vortex structures which rotate about a common axis [14]. Closer to the jet outlet, the flow is irregular and unsteady, influenced by the bow shock and a series of upright wake vortices, which shed from the leeward barrel shock.

The overall influence of this vortex is to entrain fluid from near the plate, causing an extended region of low pressure far downstream of the jet outlet. This behaviour is consistent with previous studies, and is not strongly influenced by the laminar in-flow boundary layer. The vortex is in closer proximity to the plate when compared with jets in supersonic crossflow with the same momentum ratio $(J)$, due to the higher crossflow velocity.

\section{F. Wake vortices}

The final vortex system present in the jet-in-crossflow interaction is the wake vortices. These are upright vortical structures present in the wake region of the instantaneous flow identified in Figure 14. Figure 23 shows streamlines 
(a)

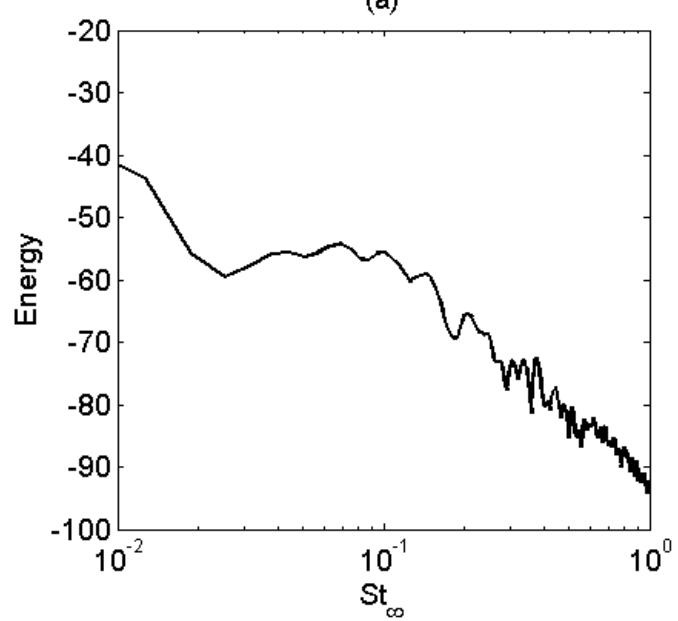

(b)

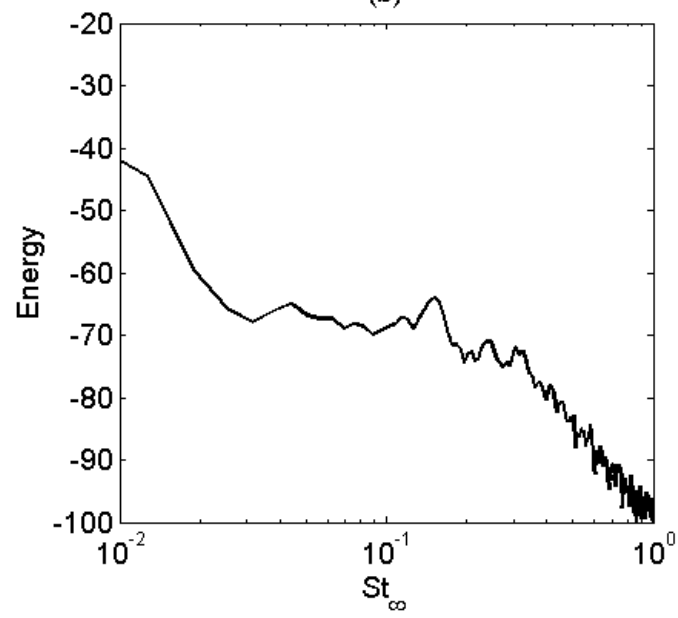

FIG. 22: Spectra of pressure measured on the flat plate $(y=0)$, on the jet centre-line (a) 5 jet diameters upstream, and (b) 20 jet diameters downstream.

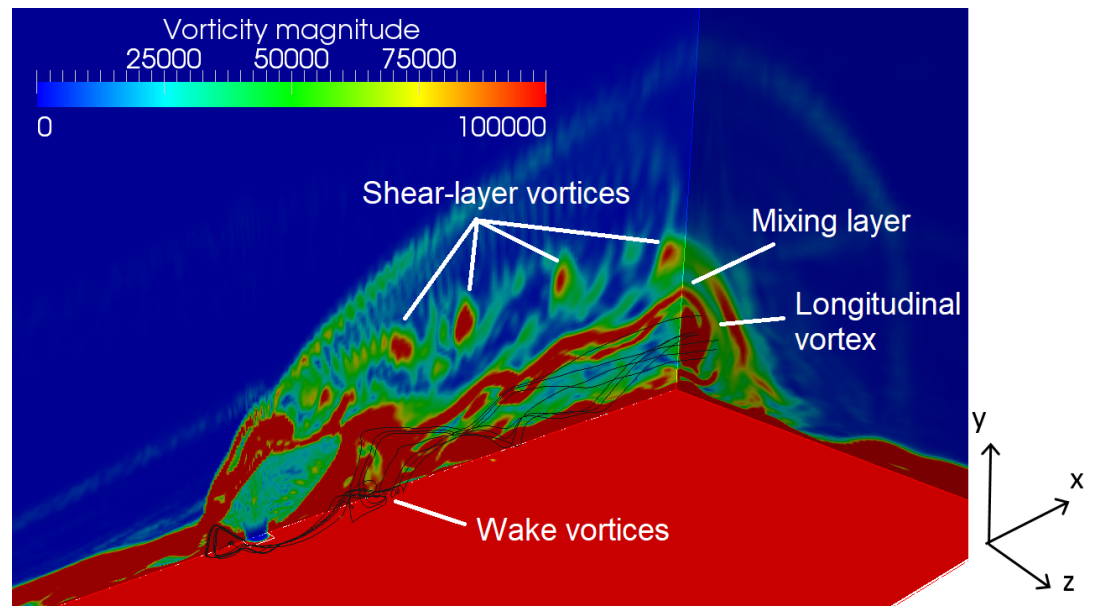

FIG. 23: Streamlines overlaid on instantaneous vorticity magnitude, showing wake vortices.

of flow first being entrained within the horseshoe vortex (Vortex A), then passing laterally around the jet outlet, before being entrained within wake vortices and lifted into the forming stream-wise counter-rotating vortex. These streamlines are overlaid on contours of instantanoues voriticity magnitude, which shows that the stream-wise vortex is located below a mixing layer, which is located below the shear-layer vortices. This mixing layer formation was observed in previous studies of supersonic jets in crossflow [5,62]. In the stream-wise direction, wake vortices form between the leeward barrel shock and the re-compression shock, and are most prominent when the jet fluid has completed most of its turning. Additionally, no upright vortices are observed near the jet outlet. These observations agree with previous descriptions [26]. In the simulation, wake vortices develop at $S t_{\infty} \approx 1-1.5$, and are convected downstream with a velocity of approximately $0.5 U_{\infty}$. As shown in Figures 14 and 23, upright wake vortices are not observed for $x / d_{j}>15$, where the fully-formed stream-wise counter-rotating vortex dominates the flow-field. In addition, wake vortices are an unsteady phenomenon, and are not present in the time-averaged flowfield. Wake vortices are located in a region of low pressure, and do not significantly impact the control force.

\section{G. Shock structure}

Figure 10 shows the time-averaged shock structure, consisting of a bow shock, barrel shocks, a separation shock, and a Mach disk. A successive series of instantaneous shock structure images are provided in Figure 24. The re-compression shock downstream is unsteady and is not present in the time-averaged flow-field. The downstream reflected shock can 

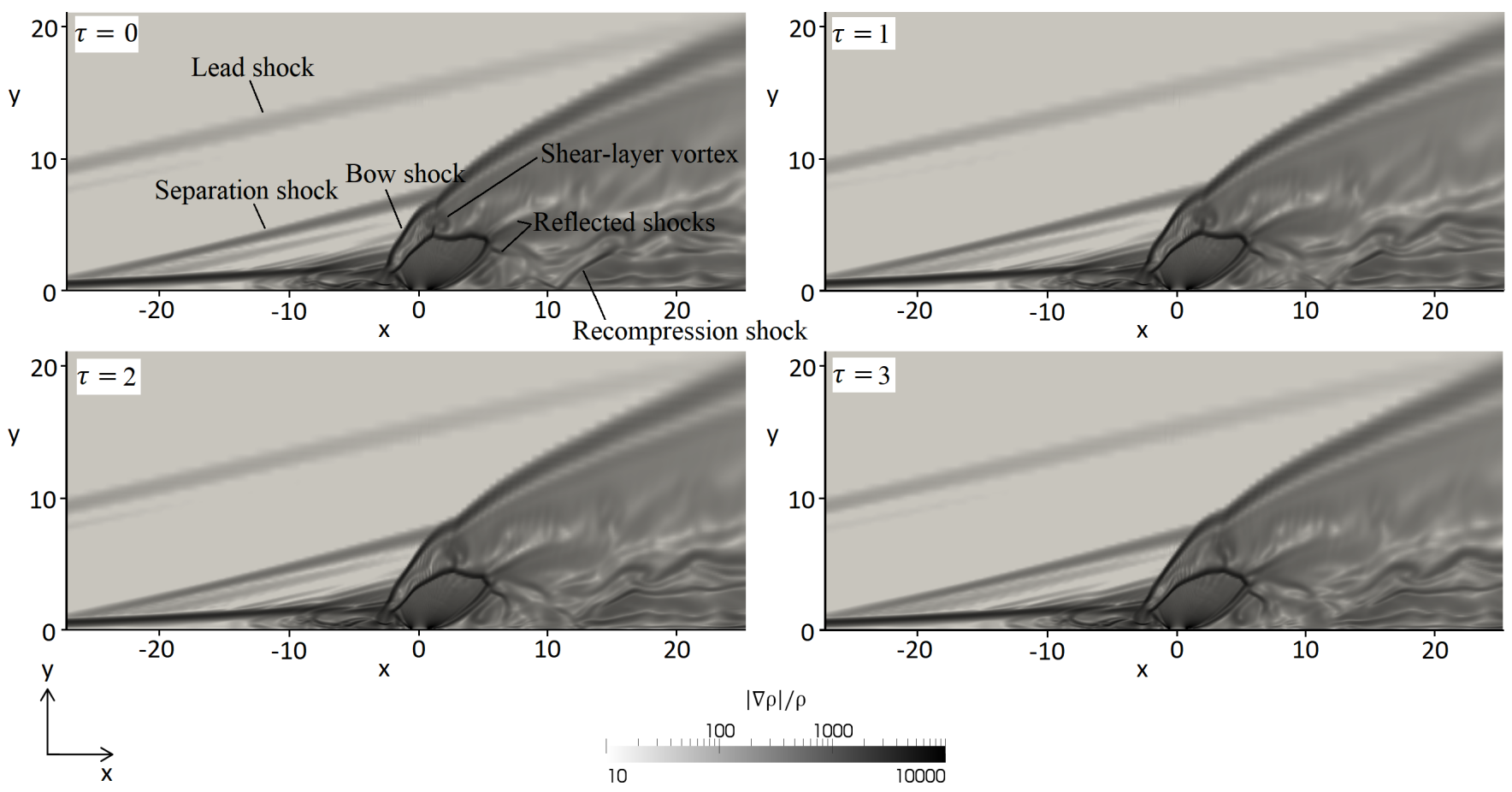

FIG. 24: Density gradient contours in the plane of symmetry, showing temporal variations in the shock structure.

be seen to extend from the triple point, where the barrel shock intersects the Mach disk, and impinges on the plate. In contrast to results at lower Mach numbers [14], a short portion of the upstream reflected shock is also visible. The bow shock is the strongest shock in the flow, and occurs at the shear-layer between the jet and the free-stream, just upstream of the windward barrel shock. As shown in Figure 24, the bow shock is periodically perturbed by shear-layer vortex shedding, as the shear-layer vortices form at the edge of the boundary layer between the bow shock and the barrel shock. With a hypersonic crossflow, the bow shock is in close proximity to the windward barrel shock. As shear-layer vortices form in the small space between the two shocks, both shocks deform concurrently. The formation of the shear-layer vortices can be seen in Figure 21, which shows an identical time-series to Figure 24 for $0 \leq \tau \leq 3$. Bow shock perturbations have been reported previously [16], but the outward motion of this bow shock perturbation is previously unreported. The deformation of the bow shock is matched by a flattening of the windward barrel shock, which was observed by Santiago and Dutton [35], and is clearly observed in Figure 24 at time $\tau=0$. The perturbation travels downstream along both the bow shock and the windward barrel shock as the shear-layer vortex convects downstream. A small local shock is also formed, and extends vertically from the sharp intersection of the two straight sections of the deformed windward barrel shock. This local shock also moves downstream with the shear-layer vortex.

During this process, the size of the Mach disk varies. The deformation of the windward barrel shock forces the two triple points closer together, and shortens the Mach disk. The Mach disk height, measured from the plate, also changes and there are corresponding changes in the leeward barrel shock, although this is much steadier and weaker than the windward barrel shock. As shown in Figure 25, two distinct regions of pressure variation correspond to the motion of the bow shock and windward barrel shock as shear-layer vortices are shed. As the location of the barrel shock and bow shock diverge at larger distances from the jet outlet, the two distinct regions of high variation become apparent. This distinction is also replicated in the pressure variation on the plate (see Figure 16 (a)), where the large variations in pressure upstream of the jet outlet splits into two separate components. The variations are caused by the changes in location of the bow shock and windward barrel shocks, as the shear-layer vortices shed.

Another clearly observed feature of the flow is the motion of the reflected shocks. The size, strength and location of both the windward and leeward reflected shocks change due to the deformation of the windward barrel shock and changes to the Mach disk. This behaviour also appears cyclic, as shear-layer vortices are periodically shed.

Close to the jet outlet, the shape of the barrel shocks is steady due to the steady jet in-flow condition. The crossflow has a negligible impact on the jet flow in the immediate vicinity of the jet outlet. Downstream of the jet, the re-compression shock is clearly present in Figure 24 at $\tau=0$, but is disturbed by the wake flow, and not present at other times, or in the time-averaged flow. 


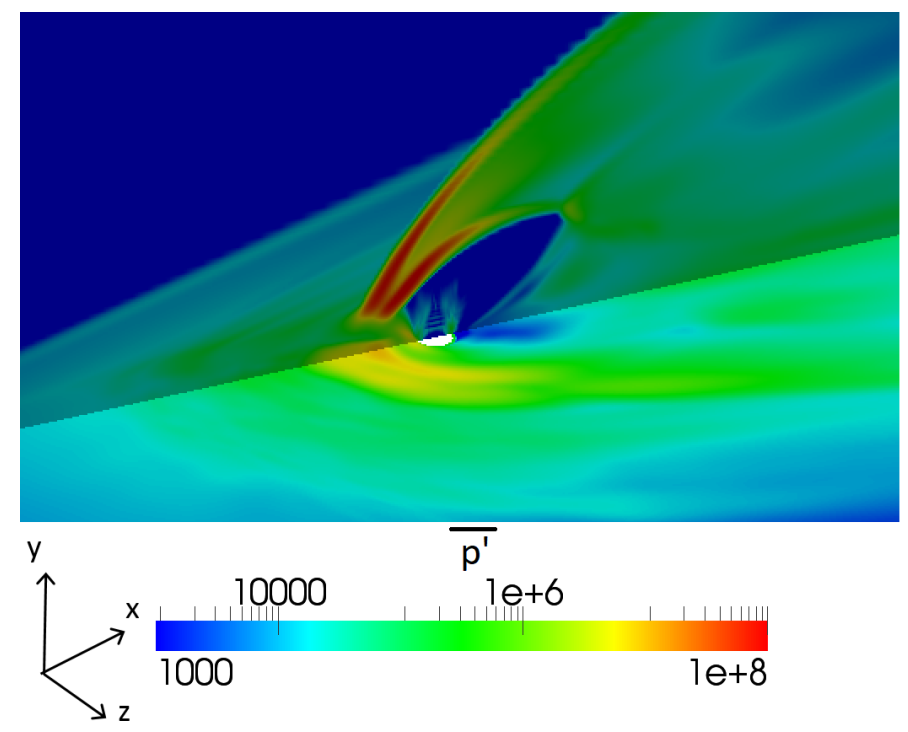

FIG. 25: Mean-square pressure variations $\left(\overline{p^{\prime} p^{\prime}}\right)$ in the plane of symmetry, due to perturbations in the bow shock and barrel shock, caused by periodic shear-layer vortex shedding.

\section{CONCLUSIONS}

In this study, the interaction between a sonic jet and a hypersonic crossflow over a flat plate with a laminar boundary layer at high jet-to-crossflow momentum ratio has been investigated. The unsteady and time-averaged behaviour of shock and vortex structures in this regime is important, as the dimensions and motion of these structures influence the pressure distribution and control force produced by a reaction control jet. The results reveal significantly different behaviour of both the horseshoe vortices and shear-layer vortices in comparison to previous studies, where turbulent in-flow boundary layers and lower freestream Mach numbers were used. The high free-stream Mach number also causes periodic deformation of the bow shock not previously observed. Other shock structures and wake vortices show similar behaviour to previous studies. The locations of the structures and their influence on the control force has been detailed.

The laminar in-flow boundary layer causes a complex horseshoe vortex structure to form in the large region of separated flow upstream of the jet. The horseshoe vortex structure consists of a quasi-steady re-circulation vortex directly upstream of the jet exit, two pairs of co-rotating vortices which periodically coalesce, and an additional quasi-steady vortex. This structure results in a large low pressure region upstream of the jet outlet.

There is a high pressure region immediately upstream of the jet outlet which wraps around the jet outlet. The pressure is highly unsteady in this region, and variations are driven by the motion of the horseshoe vortex structure, and the movement of the bow shock and barrel shock, corresponding to shear-layer vortex shedding. The development of shear-layer vortices is delayed by the thicker laminar boundary layer in comparison with previous studies, where a thinner turbulent boundary layer was present. Once formed, these vortices shed at lower frequency than in lower Mach number flows, and convect far downstream causing fast moving, localised high pressure regions on the plate.

A complex and unstructured series of wake vortices is observed in the region between the leeward barrel shock and the re-compression shock. Wake vortices form in a region of low pressure and have little influence on the control force.

The close proximity of the bow shock to the windward barrel shock means that the bow shock is deformed by the development and convection of shear-layer vortices. Periodic deformation of the windward barrel shock and formation of a local shock matches previous observations; however, the subsequent deformation of the bow shock has not previously been reported.

[1] Bolender, M. A. and Doman, D. B., "Nonlinear Longitudinal Dynamical Model of an Air-breathing Hypersonic Vehicle," AIAA Journal of Spacecraft and Rockets, Vol. 44, No. 2, 2007, pp. $374-387$.

[2] Chavez, F. R. and Schmidt, D. K., "Flight Dynamics and Control of Elastic Hypersonic Vehicles Modeling Uncertainties," $A I A A$, Vol. Paper 3629-94, 1994, pp. $766-774$. 
[3] Mei, C., Abdel-Motagaly, K., and Chen, R., "Review of Nonlinear Panel Flutter At Supersonic and Hypersonic Speeds," Applied Mechanics Reviews, Vol. 52, No. 10, 1999, pp. 321 - 332.

[4] Margason, R. J., "Fifty Years of Jet in Cross Flow Research," Computational and Experimental Assessment of Jets in Cross Flow, AGARD-CP-534, Advisory Group for Aerospace Research and Development, Winchester, UK, 1993.

[5] Mahesh, K., "The Interaction of Jets with Crossflow," Annual Review of Fluid Mechanics, Vol. 45, 2013 , pp. 379 - 407.

[6] Spaid, F. W. and Zukoski, E. E., "A Study of the Interaction of Gaseous Jets from Transverse Slots with Supersonic External Flows," AIAA Journal, Vol. 6, No. 2, 1968, pp. $205-212$.

[7] Schetz, J. A., Weinraub, R. A., and Mahaffey, R. E., "Super-sonic Transverse Injection into a Supersonic Stream," AIAA Journal, Vol. 6, 1968, pp. 993, 994.

[8] Rogers, R. C., "A study of the mixing of hydrogen injected normal to a supersonic airstream," Tech. rep., NASA TN D-6114, 1971.

[9] Rana, Z. A., Thornber, B., and Drikakis, D., "Transverse jet injection into a supersonic turbulent cross-flow," Physics of Fluids, Vol. 23, 2011.

[10] Beresh, S. J., Henfling, J. F., Erven, R. J., and Spillers, R. W., "Penetration of a Transverse Supersonic Jet into a Subsonic Compressible Crossflow," AIAA Journal, Vol. 43, No. 2, 2005, pp. 379 - 389.

[11] Kelso, R. M., Lim, T. T., and Perry, A. E., "An experimental study of round jets in cross-flow," Journal of Fluid Mechanics, Vol. 306, 1996, pp. 111-144.

[12] Krothapalli, A., Lourenco, L., and Buchlin, J. M., "Separated Flow Upstream of a Jet in a Crossflow," AIAA Journal, Vol. 28, No. 3, 1990, pp. $414-420$.

[13] Ruiz, A. M., Lacaze, G., and Oefelein, J. C., "Flow topologies and turbulence scales in a jet-in-cross-flow," Physics of Fluids, Vol. 27, 2015.

[14] Viti, V., Neel, R., and Schetz, J. A., "Detailed Flow Physics of the Supersonic Jet Interaction Flow Field," Physics of Fluids, Vol. 21, 2009.

[15] Chenault, C. F. and Beran, P. S., " $k-\epsilon$ and Reynold's stress turbulence model comparisons for two-dimensional injection flows," AIAA Journal, Vol. 36, No. 8, 1998, pp. 1401.

[16] Kawai, S. and Lele, S. K., "Dynamics and mixing of a sonic jet in a supersonic turbulent crossflow," Center for Turbulence Research Annual Research Briefs, pp. 285 - 98, Stanford CA, 2009.

[17] Fric, T. F. and Roshko, A., "Vortical structure in the wake of a transverse jet," Journal of Fluid Mechanics, Vol. 279, 1994 , pp. $1-47$.

[18] Yuan, L. L., Street, R. L., and Ferziger, J. H., "Large-eddy simulations of a round jet in crossflow," Journal of Fluid Mechanics, Vol. 379, 1999, pp. $71-104$.

[19] Cortelezzi, L. and Karagozian, A. R., "On the Formation of the Counter-Rotating Vortex Pair in Transverse Jets," Journal of Fluid Mechanics, Vol. 446, 2001, pp. $347-373$.

[20] Lim, T. T., New, T. H., and Lou, S. C., "On the development of large-scale structures of a jet normal to a cross flow," Physics of Fluids, Vol. 13, 2001, pp. $770-775$.

[21] Smith, S. H. and Mungal, M. G., "Mixing, structure and scaling of the jet in crossflow," Journal of Fluid Mechanics, Vol. 357, 1998, pp. $83-122$.

[22] Getsinger, D. R., Gevorkyan, L., Smith, O. I., and Karagozian, A. R., "Structural and stability characteristics of jets in crossflow," Journal of Fluid Mechanics, Vol. 760, 2014, pp. 342 - 367.

[23] Karagozian, A. R., "The jet in crossflow," Physics of Fluids, Vol. 26, 2014.

[24] Gevorkyan, L., Shoji, T., Getsinger, D. R., Smith, O. I., and Karagozian, A. R., "Transverse jet mixing characteristics," Journal of Fluid Mechanics, Vol. 790, 2016, pp. $237-274$.

[25] Chai, X., Iyer, P. S., and Mahesh, K., "Numerical study of high speed jets in crossflow," Journal of Fluid Mechanics, Vol. 785, 2015, pp. $152-188$.

[26] van Lerberghe, W. M., Santiago, J. G., Dutton, J. C., and Lucht, R. P., "Mixing of a Sonic Transverse Jet Injected into a Supersonic Flow," AIAA Journal, Vol. 38, No. 3, 2000, pp. $470-479$.

[27] Ben-Yakar, A., Experimental Investigation of Mixing and Ignition of Transverse Jets in Supersonic Crossflows, Ph.D. thesis, Stanford University, Department of Mechanical Engineering, 2000.

[28] Andre, T., Durant, A., and Fedioun, I., "Numerical Study of Supersonic Boundary-Layer Transition due to Sonic Wall Injection," AIAA Journal, Vol. 55, No. 5, 2017, pp. 1530 - 1547.

[29] Sau, R. and Mahesh, K., "Optimization of pulsed jets in crossflow," Journal of Fluid Mechanics, Vol. 653,2010, pp. 365 -390 .

[30] Gruber, M. R., Nejad, A. S., Chen, T. H., and Dutton, J. C., "Large structure convection velocity measurements in compressible transverse injection flowfields," Experiments in Fluids, Vol. 22, 1997, pp. 397 - 407.

[31] Ben-Yakar, A., Mungal, M. G., and Hanson, R. K., "Time evolution and mixing characteristics of hydrogen and ethylene transverse jets in supersonic crossflows," Physics of Fluids, Vol. 18, 2006.

[32] Takahashi, H., Masuya, G., and Hirota, M., "Effects of Injection and Main Flow Conditions on Supersonic Turbulent Mixing Structure," AIAA Journal, Vol. 48, No. 8, 2010, pp. 1748 - 1756.

[33] Watanabe, J., Kouchi, T., Takita, K., and Masuya, G., "Large-Eddy Simulation of Jet in Supersonic Crossflow with Different Injectant Species," AIAA Journal, Vol. 50, No. 12, 2012, pp. $2765-2778$.

[34] Watanabe, J., Kouchi, T., Takita, K., and Masuya, G., "Characteristics of Hydrogen Jets in Supersonic Crossflow: LargeEddy Simulation Study," AIAA Journal of Propulsion and Power, Vol. 29, No. 3, 2013, pp. 661 - 674.

[35] Santiago, J. G. and Dutton, J. C., "Velocity Measurements of a Jet Injected into a Supersonic Crossflow," Journal of Propulsion and Power, Vol. 13, No. 2, 1997, pp. $264-273$. 
[36] Kawai, S. and Lele, S. K., "Large-Eddy Simulation of Jet Mixing in Supersonic Crossflows," AIAA Journal, Vol. 48, No. 9, 2010, pp. $2063-2083$.

[37] Weller, H., Tabor, G., Jasak, H., and Fureby, C., "A tensorial approach to computational continuum mechanics using object-oriented techniques," Computers in Physics, Vol. 12, No. 6, 1998, pp. 620 - 631.

[38] Greenshields, C. J., Weller, H. G., Gasparini, L., and Reese, J. M., "Implementation of semi-discrete, non-staggered central schemes in a colocated, polyhedral, finite volume framework for high-speed viscous flows," International Journal for Numerical Methods in Fluids, Vol. 63, 2010, pp. 1 - 21.

[39] Kurganov, A., Noelle, S., and Petrova, G., "Semidiscrete Central-Upwind Schemes for Hyperbolic Conservation Laws and Hamilton-Jacobi Equations," SIAM Journal of Scientific Computing, Vol. 23, No. 3, 2001, pp. 707 - 740.

[40] van Leer, B., "Towards the Ultimate Conservative Difference Scheme II. Monotonicity and Conservation Combined in a Second Order Scheme," Journal of Computational Physics, Vol. 14, 1974, pp. 361 - 370.

[41] Jasak, H., Error Analysis and Estimation for the Finite Volume Method with Applications to Fluid Flows, Ph.D. thesis, Imperial College of Science, Technology and Medicine, Department of Mechanical Engineering, 1996.

[42] Fureby, C., "On subgrid scale modeling in large eddy simulation of compressible fluid flow," Physics of Fluids, Vol. 8, 1996, pp. $1301-1311$.

[43] Grinstein, F. F. and Fureby, C., "On Flux-Limiting-Based Inplicit Large Eddy Simulation," Journal of Fluids Engineering, Vol. 129, 2007, pp. $1483-1492$.

[44] Grinstein, F. F., Fureby, C., and DeVore, C. R., "On MILES based on flux-limiting algorithms," International Journal for Numerical Methods in Fluids, Vol. 47, 2005, pp. 1043 - 1051.

[45] Grinstein, F. F., Margolin, L., and Rider, W., Implicit Large Eddy Simulation: Capturing the Turbulent Flow Dynamics, Cambridge University Press, 2005.

[46] Roe, P. L., "Characteristic-Based Schemes for the Euler Equations," Annual Review of Fluid Mechanics, Vol. 18, 1986, pp. $337-365$.

[47] Miller, W. A., Medwell, P. R., Kim, M., and Doolan, C. J., "Computational Methodology for Investigating the Transient Interaction between a Reaction Control Jet and a Hypersonic Crossflow," AIAA SciTech Forum, 4 - 8 January 2016, San Diego, California, USA, 54th AIAA Aerospace Sciences Meeting, 2016.

[48] Erdem, E., Active Flow Control Studies at Mach 5: Measurement and Computation, Ph.D. thesis, University of Manchester, School of Mechanical, Aerospace and Civil Engineering, 2011.

[49] Viti, V., Numerical studies of the jet interaction flowfield with a main jet and an array of smaller jets., Ph.D. thesis, Virginia Tech, Department of Aerospace and Ocean Engineering, 2002.

[50] Erdem, E. and Kontis, K., "Numerical and Experimental Investigation of Transverse Injection Flows," Shock Waves, Vol. 20, 2010, pp. $103-118$.

[51] Fureby, C., "Large Eddy Simulation: A Useful Tool for Engineering Fluid Dynamics," 18th Australasian Fluid Mechanics Conference, Launceston, Australia, 3 - 7 December, 2012.

[52] Pope, S. B., Turbulent Flows, Cambridge University Press, 2000.

[53] Li, L., Hirota, M., Ouchi, K., and Saito, T., "Evaluation of fluidic thrust vectoring nozzle via thrust pitching angle and thrust pitching moment," Shock Waves, Vol. 27, 2017, pp. 53 - 61.

[54] Roache, P. J., "Quantification of Uncertainty in Computational Fluid Dynamics," Annual Review of Fluid Mechanics, Vol. 29, 1997, pp. $123-160$.

[55] Popinski, Z. and Ehrlich, C. F., "Development Design Methods for Predicting Hypersonic Dynamic Control Characteristics," Tech. rep., US Air Force Flight Dynamics Laboratory, AFFDL-TR-66-85, 1966.

[56] Erdem, E. and Kontis, K., "Non-Plasma and Plasma Transverse Jets in Hypersonic Crossflow," 16th AIAA/DLR/DGLR International Space Planes and Hypersonic Systems Technologies Conference, Bremen, Germany, 2009.

[57] Vlagov, V. V., Masyakin, N. E., and Polyanskii, M. N., "Penetration Depth of a Jet Injected into an Oncoming Supersonic Flow," Fluid Dynamics, Vol. 15, 1967, pp. $599-602$.

[58] Rothstein, A. D. and Wantuck, P. J., "A study of the normal injection of hydrogen into a heated supersonic flow using planar laser-induced fluorescence," 28th SAE, ASME, ASEE Joint Propulsion Conference and Exhibit, Nashville, TN, 1992.

[59] Vranos, A. and Nolan, J. J., "Supersonic mixing of a light gas and air," AIAA Joint Propulsion Conference, Colorado Springs, CO, 1965.

[60] McDaniel, J. C. and Graves, J., "Laser-induced fluorescence visualization of transverse gaseous injection in a nonreacting supersonic combustor," Journal of Propulsion and Power, Vol. 4, 1988, pp. 591 - 597.

[61] Cassel, L. A., "Applying Jet Interaction Technology," AIAA Journal of Spacecraft and Rockets, Vol. 40, No. 4, 2003, pp. $523-537$.

[62] Chai, X. and Mahesh, K., "Simulation of High Speed Turbulent Jets in Crossflows," AIAA Aerospace Sciences Meeting, Orlando, FL, 2011. 